\title{
Concepts for Enhanced Energy Absorption Using Hollow Micro-Lattices
}

\section{Citation}

Evans, A. G., M. Y. He, V. S. Deshpande, John W. Hutchinson, A. J. Jacobsen, and W. BarvosaCarter. 2010. Concepts for enhanced energy absorption using hollow micro-lattices. International Journal of Impact Engineering 37(9): 947-959.

\section{Published Version}

doi:10.1016/j.ijimpeng.2010.03.007

\section{Permanent link}

http://nrs.harvard.edu/urn-3:HUL.InstRepos:4211042

\section{Terms of Use}

This article was downloaded from Harvard University's DASH repository, and is made available under the terms and conditions applicable to Open Access Policy Articles, as set forth at http:// nrs.harvard.edu/urn-3:HUL.InstRepos:dash.current.terms-of-use\#OAP

\section{Share Your Story}

The Harvard community has made this article openly available.

Please share how this access benefits you. Submit a story.

Accessibility 


\title{
Concepts for Enhanced Energy Absorption Using Hollow Micro-Lattices
}

\author{
A. G. Evans, M. Y. He, \\ Materials Department, College of Engineering, UCSB, CA, 93106 \\ V. S. Deshpande, \\ Engineering Department, Cambridge University, Trumpington St, Cambridge, CB2 \\ 1PZ, UK \\ J. W. Hutchinson, \\ School of Engineering and Applied Sciences, Harvard University, Cambridge, MA \\ 02138
}

\author{
A. J. Jacobsen, W. Barvosa-Carter, \\ HRL Laboratories, Malibu, CA, 90265
}

\begin{abstract}
We present a basic analysis that establishes the metrics affecting the energy absorbed by multilayer cellular media during irreversible compaction on either a mass or volume basis. The behaviors at low and high impulse levels are distinguished through the energy dissipated in the shock. The overall mass of an energy absorbing system (comprising a cellular medium and a buffer) is minimized by maximizing the non-dimensional dissipation per unit mass parameter for the cellular medium, $\Lambda \equiv U_{m} \rho_{s} / \sigma_{Y}$, where $U_{m}$ is the dissipation per unit mass of the cellular medium, ascertained from the area under the quasi-static compressive stress/strain curve, $\sigma_{Y}$ the yield strength of the constituent material and $\rho_{s}$ the density of the material used in the medium. Plots of $\Lambda$ against the non-dimensional stress transmitted through the medium, $\sigma_{t r} / \sigma_{Y}$ demonstrate the relative energy absorbing characteristics of foams and prismatic media, such as honeycombs. Comparisons with these benchmark systems are used to demonstrate the superior performance of micro-lattices, especially those with hollow truss members. Numerical calculations demonstrate the relative densities and geometric configurations wherein the lattices offer benefit. Experimental results obtained for a $\mathrm{Ni}$ micro-lattice with hollow members not only affirm the benefits, but also demonstrate energy absorption levels substantially exceeding those predicted by analysis. This assessment highlights the new opportunities that tailored micro-lattices provide for unprecedented levels of energy absorption for protection from impulsive loads.
\end{abstract}

Keywords: Energy absorption, impulse, cellular medium, micro-lattices, finite element. 


\section{Nomenclature}

\begin{tabular}{|c|c|}
\hline $\mathrm{E}$ & Young's modulus \\
\hline $\mathrm{E}_{\mathrm{t}}$ & tangent modulus after plastic yielding \\
\hline$\varepsilon_{D}$ & strain at densification \\
\hline$\varepsilon_{Y}$ & yield strain for the constituent material \\
\hline$h$ & thickness of the crushable medium \\
\hline$h_{\min }$ & minimum thickness of the cellular medium \\
\hline $\mathrm{KE}$ & kinetic energy \\
\hline$I$ & impulse per area \\
\hline$I_{\text {trans }}$ & transmitted impulse per unit area \\
\hline$\Lambda$ & non-dimensional dissipation per mass parameter \\
\hline M & momentum per area \\
\hline$m_{b}$ & mass per area of the planar buffer \\
\hline$m_{\text {total }}^{\min }$ & minimum mass \\
\hline $\mathrm{n}$ & exponent in the crushing strength formula \\
\hline$p_{0}$ & overpressure with associated impulse per area, $I$ \\
\hline \multicolumn{2}{|c|}{$\Pi \equiv h \rho_{s} \bar{\rho} \varepsilon_{D} / m_{b}$} \\
\hline $\bar{\rho}$ & $\begin{array}{l}\text { Ratio of the mass of the cellular medium to that for the buffer } \\
\text { relative density of the cellular material. }\end{array}$ \\
\hline$\rho_{s}$ & density of the material used in the medium \\
\hline$\sigma_{\text {peak }}$ & initial peak stress, associated with member buckling \\
\hline$\sigma_{p l}$ & unidirectional crushing stress, $\sigma_{p l}$, with strain at densification, $\varepsilon_{D}$ \\
\hline$\sigma_{t r}$ & stress transmitted through the medium \\
\hline$\sigma_{t h}$ & damage threshold \\
\hline$\sigma_{Y}$ & yield strength of the constituent material \\
\hline$U_{m}$ & quasi-static energy absorbed per unit mass of the cellular medium \\
\hline$V_{\text {total }}$ & volume of the system \\
\hline$\Psi \equiv \frac{\sigma_{p l} m_{b}^{2}}{M^{2} \rho_{s} \bar{\rho}}$ & Ratio of the static to the dynamic strength of the cellular medium \\
\hline
\end{tabular}

The HRL micro-lattice
A
cross-sectional area of pyramidal truss members
$\mathrm{L} \quad$ length of members
$\mathrm{R} \quad$ radius of members
$\mathrm{t} \quad$ thickness of members
$\theta \quad$ included angle between member and base 


\section{Introduction}

Concepts for protecting structures from impulsive loads are of current interest. The prototypical threat comprises a shock in air characterized by an overpressure $p_{0}$ with associated impulse/area, I. For protection against the overpressure, an intervening medium is required that reduces the pressure from $p_{0}$ to a level below a damage threshold, $\sigma_{t h}$. To achieve this, the medium must be capable of large volume decrease at constant pressure (Figure 1) [1, 2] (thereupon extending the impulse duration). Dense solids and fluids are not suitable because they are nearly incompressible. Appropriate materials include low-density cellular solids such as reticulated polymers [3], metal foams [1], pre-crushed honeycombs [3] and lattice solids [4], all with relative density, $\bar{\rho}$, in the $0.1-10 \%$ range and densification strains preferably exceeding $80 \%$. Rectification is required by means of a planar buffer, mass/area $m_{b}$, positioned to face the shock (with the compressible medium attached) [1, 2]. The buffer acquires a momentum per area, $M$, equal to the transmitted impulse per unit area, $I_{\text {trans }}$, with associated kinetic energy/area, $K E=M^{2} / 2 m_{b}$ [1]. This kinetic energy must either be dissipated by plastic deformation or stored elastically in the medium. The research objective in this area is to seek a medium that dissipates this $\mathrm{KE}$ at lowest possible mass/area, while not transmitting a stress greater than $\sigma_{t h}$-a design constraint dictated by the nature of the structure being protected. We examine the potential of all known classes of cellular materials for attaining this objective and reveal a new regime wherein energy absorption per unit mass is maximized by using hollow micro-lattices, a consequence of their favorable topological characteristics.

While cellular materials such as stochastic foams and honeycomb are readily available, fabrication of appropriate micro-lattice structures has been challenging. As a consequence, experimental investigation of their energy absorption capability has been limited to solid truss geometries [5] or hollow-tube geometries [6] with discontinuous node connectivity. Recent developments have provided a new fabrication capability for realizing micro-lattices with unit cells in the 0.1 to $>10 \mathrm{~mm}$ range (Figure 2a) [7-9]. These structures have nearly-ideal, truss-like geometries with linear truss members, well connected nodes and smooth surfaces. Subsequent electro-deposition and removal of the base polymer allows conversion to metallic hollow tube geometries (Figure 2b). This method allows for relatively straightforward formation of $\sim 1-2 \mathrm{~cm}$ thick structures. Herein, such media are referred to as HRL micro-lattices. Given the beneficial postbuckling response of hollow (rather than solid) tubes [10-16], the ensuing energy absorption assessment emphasizes the attributes of hollow micro-truss lattices.

For ranking micro-lattices relative to other cellular media, a set of metrics is required. These are presented in the next section. They are based on the minimum thickness of the cellular medium, $h_{\min }$, needed to absorb the KE of the buffer which, in turn, dictates the

minimum mass, $m_{\text {total }}^{\min }$, or volume $V_{\text {total }}$ of the system. This assessment is followed by benchmark results for well-characterized conventional systems comprising foams and prismatic lattices, such as honeycombs [3]. Thereafter, the potential for superior 
performance is examined by analyzing the response of micro-lattices. While the assessment is strictly applicable to multilayer cellular media that experience layer-bylayer sequential dynamic crushing, some of the energy absorption mechanisms are elucidated through simulations and measurements conducted on single layer microlattices.

\section{Metrics.}

The Minimum Thickness of the Cellular Medium. The objective is to provide an analysis of the dynamic crushing of a cellular medium attached to a buffer. The medium is presumed to deform with "ideal" mechanical response (Figures 1 and 3a), characterized by a constant unidirectional crushing stress, $\sigma_{p l}$, with strain at densification, $\varepsilon_{D}$. The major features governing the minimum thickness of the cellular medium, $h_{\min }$, are presented in detail in the Appendix and with two non-dimensional parameters governing the response:

- Ratio of the mass of the cellular medium to that for the buffer, $\Pi \equiv h \rho_{s} \bar{\rho} \varepsilon_{D} / m_{b}$, where $h$ is the thickness of the crushable medium

- Ratio of the static to the dynamic strength of the cellular medium, $\Psi \equiv \frac{\sigma_{p l} m_{b}^{2}}{M^{2} \rho_{s} \bar{\rho}}$

where $\rho_{s}$ is the density of the parent material and $\bar{\rho}$ the relative density of the cellular material. Based on the analysis, two regimes have been ascertained based on $\Pi$ : regime $I$ is essentially quasi-static while regime $I I$ is dynamic (Figure 4).

Regime I: When $\Pi \leq 2$, shock effects in the medium are minimal, and the maximum dissipation/area is quasi-static, with $U=\sigma_{p l} \varepsilon_{D} h$. Equating the dissipation to the kinetic energy gives the minimum thickness to arrest the buffer as:

$$
h_{\min }=\frac{M^{2}}{2 m_{b} \sigma_{p l} \varepsilon_{D}}
$$

or, in terms of the foregoing non-dimensional parameters,

$$
\Pi_{\min }=1 / 2 \Psi
$$

where $\Pi_{\min } \equiv h_{\min } \rho_{s} \bar{\rho} \varepsilon_{D} / m_{b}$.

Provided that the actual thickness, $h$, exceeds $h_{\min }$, the pressure imparted to the structure does not exceed $\sigma_{p l}$. Accordingly, by choosing $\sigma_{p l}<\sigma_{t h}$ the structure is protected. When $h<h_{\min }$, the medium densifies before the buffer arrests and much larger pressures are transmitted when the buffer "slaps" into the structure. In regime I, the minimum 
thickness decreases as the buffer mass/area increases (1a) leading to a minimum in the total mass, elaborated below.

Regime II. When $\Pi \geq 2$, a shock develops in the medium. Because of the density and stress discontinuity across the shock, additional energy is absorbed [8,9]. The minimum thickness (Figure 4) is smaller than that estimated by extrapolating from regime I and given by

$$
\Pi_{\min } \approx 1 / \sqrt{\Psi}
$$

or, in dimensional terms:

$$
h_{\min } \approx \frac{M}{\sqrt{\rho_{s} \bar{\rho} \sigma_{p l} \varepsilon_{D}}}
$$

Now, the minimum thickness of the cellular medium is independent of the mass per unit area of the buffer as elaborated below and in the Appendix.

The Minimum Mass for the System. The system mass is dependent on the impulse in accordance with the foregoing two regimes. In regime I, a minimum exists in the total (buffer and cellular medium) mass per area to mitigate the overpressure (Appendix) given by:

$$
\begin{aligned}
& m_{\text {total }}^{\text {min }}=M \sqrt{\frac{2 \bar{\rho} \rho_{s}}{\sigma_{p l} \varepsilon_{D}}} \\
& \equiv M \sqrt{\frac{2}{U_{m}}}
\end{aligned}
$$

where $U_{m} \equiv \sigma_{p l} \varepsilon_{D} / \rho_{s} \bar{\rho}$ is the quasi-static energy absorbed per unit mass of the cellular medium. At higher impulse levels, in regime II, the total mass is (Appendix)

$$
m_{\text {total }}=m_{b}+M \sqrt{1 / U_{m}}
$$

While there is no minimum, if the buffer mass is selected to satisfy the inequality

$$
m_{b} \leq(\sqrt{2}-1) M \sqrt{1 / U_{m}}
$$

the lowest weight system resides in regime II. Accordingly, maximizing $U_{m}$ always minimizes the overall weight. This is achieved by selecting constituents with lowest possible density, configured within architectures that experience "ideal" plateau response up to large densification strains. 
Non-Ideal Crushing. Among the available cellular media, many compress with an initial peak, $\sigma_{\text {peak }}$, associated with member buckling, followed by an oscillating stress/strain response as the medium compresses (Figure 3b) [3]. This response is undesirable because the stress transmitted through the medium is $\sigma_{\text {peak }}$, while the dissipation occurs at a lower stress. Consequently, the objective is to seek media with lowest possible density, $\rho_{s} \bar{\rho}$, that compress to a large densification strain, $\varepsilon_{D}$ without a significant initial peak.

Media with Yield Governed Crushing. When members yield before buckling, the nondimensional dissipation/mass parameter that distinguishes the topology from the material properties is:

$$
\Lambda=\frac{U_{m} \rho_{s}}{\sigma_{Y}} \equiv \frac{\sigma_{p l}}{\sigma_{Y}} \cdot \frac{\varepsilon_{D}}{\bar{\rho}}
$$

with $\sigma_{Y}$ the yield strength of the constituent material. The preferred topology is that providing the requisite $\sigma_{p l} / \sigma_{Y}$ at the lowest relative density. Expressing the crushing strength through a generalized power law [3];

$\sigma_{p l} / \sigma_{Y}=\zeta \bar{\rho}^{n}$

(with $n$ between 1 and 2), the dissipation becomes

$$
\Lambda=\zeta \varepsilon_{D} \bar{\rho}^{n-1}
$$

Thus, the smaller the exponent, $n$, the greater the energy absorbed/mass. This straightforward principle rationalizes the trends in energy absorption among different topologies. Note that, for lattice topologies with $n=1, \Lambda$ is essentially independent of the relative density.

By equating the loads required for elastic buckling and yielding, it has been demonstrated that truss-based cellular media are yield (rather than buckling) dominated at relative densities that approximately satisfy [3],

$$
\bar{\rho}>36 \varepsilon_{Y}^{2}
$$

where $\varepsilon_{Y}$ is the yield strain for the constituent material. For metals (with $\varepsilon_{Y} \approx 10^{-3}$ ) yielding invariably predominates. Moreover, even for polymers $\left(\varepsilon_{Y} \approx 10^{-2} \rightarrow 10^{-1}\right)$, only systems with exceptionally low $\bar{\rho}$ are buckling limited.

\section{Benchmarks}

Stochastic media such as foams are relatively isotropic. Those whose cell walls yield by plastic bending before elastically buckling have a plateau stress that scales as [3]: 
$\sigma_{p l} / \sigma_{Y} \approx 0.3 \bar{\rho}^{3 / 2}$

giving non-dimensional dissipation/mass:

$\Lambda \approx 0.3 \bar{\rho}^{1 / 2}(1-1.4 \bar{\rho})$

where we have taken $\varepsilon_{D}=1-1.4 \bar{\rho}$.

A cross plot of $\Lambda$ against the non-dimensional transmitted stress, $\sigma_{p l} / \sigma_{Y}$, characterizes all foams at all viable relative densities (Figure 5 ). Each $\bar{\rho}$ is represented by a single point on the curve, as indicated on the Figure.

Prismatic systems are anisotropic. Honeycombs are illustrative. In the transverse orientation, the plateau stress is [3]:

$\sigma_{p l} / \sigma_{Y} \approx 0.5 \bar{\rho}^{2}$

such that (Figure 5)

$\Lambda \approx 0.5 \bar{\rho}(1-1.4 \bar{\rho})$

Relative to foams, because of the larger exponent $n$, the energy absorbed at specified transmitted stress is lower.

In the axial direction, the peak stress is $[3,12]$ :

$\sigma_{\text {peak }} / \sigma_{Y} \approx 5.2 \bar{\rho}^{5 / 3}$

This peak is followed by oscillations at lower average stress, $\lambda \sigma_{\text {peak }}$, such that the energy absorption has the form:

$\Lambda \approx 5.2 \lambda \bar{\rho}^{2 / 3}(1-1.4 \bar{\rho})$

Results for $\lambda=1 / 3$ are plotted on Figure 5. The extreme anisotropy limits the use of prismatic structures when off-axis loadings are likely.

\section{Analysis of Micro-Lattices.}

Configurations and Geometric Variables. The HRL micro-lattice (Figure 2a) consists of an ensemble of pyramidal truss members of radius $R$, wall thickness $t$, length $L$ with an included angle, $\theta=60^{\circ}$ (see Fig. 2), attached at the nodes. Because the response to compression is characterized by the post-buckling deformation, the following geometric 
parameters are important: (i) The aspect ratio $A / L^{2}$ (with $L$ the member length and $A$ the cross-sectional area). (ii) The relative wall thickness, $R / t$. (iii) The orientation of the truss relative to the crush axis, $\theta$. For a system with $\theta=60^{\circ}$, the relative density is given by

$\bar{\rho}=\frac{16}{\sqrt{3}} \frac{A}{L^{2}}$

where $A \approx 2 \pi R t$

Methods and Preliminary Assessments: For tractability the analysis is restricted to a single layer lattice, with the understanding that the results can be used to predict the behavior of a multilayer by using a shock propagation analysis (Appendix). Several unit cell configurations and numerical techniques have been explored (Figure 6). Beam elements (Figure 6a) are unable to capture local plastic buckling (wrinkling). Consequently, unit cells with 3D shell elements (exemplified by the pyramidal truss Figure 6c) have been used, as well as a single member of the pyramid (Figure 6b) with symmetry boundary conditions. The commercial finite element code, ABAQUS/Explicit is used for the finite element simulations. A convergence study shows a mesh of 5000 3D shell elements (S4R, 64 (hoop) x 80 (axial)) for a single tube gives accurate results.

The calculations are conducted for a material with properties resembling Al 7076 T6: albeit that the normalizations render universality. The material is assigned bilinear stress versus strain response, with moderate strain hardening (tangent modulus, $E_{T} / E=0.01$ ), yield strain, $\varepsilon_{Y}=6 \times 10^{-3}$ and Young's modulus, $E=70 \mathrm{GPa}$. In all calculations the truss members are loaded in axial compression with fixed ends. The calculations are continued to large strains to capture densification.

Eigenmodes. Before proceeding, an eigenvalue analysis has been used to delineate the buckling modes. The analysis is elastic. Its purpose is to reveal the various competing shapes of the plastic buckling modes and not provide an estimate of the load carrying capacity. Moreover, imperfections in the shape of these eigenmodes are introduced in the analysis to ensure that realistic load-deflection behavior is triggered. Three classifications emerge: (i) global buckling, (ii) axi-symmetric and (iii) non-axisymmetric local buckling (wrinkling). These modes for the plastic buckling of tubes have previously been classified in accordance with the mechanism map depicted on Figure 7 [16].

Slender trusses: characterized by $A / L^{2}=0.01, \mathrm{R} / \mathrm{t}=3$ (for $\theta=90^{\circ}$ ) and by $\bar{\rho}=2 \%$, $A / L^{2}=0.0015, \mathrm{R} / \mathrm{t}=5$ (for $\theta=60^{\circ}$ ). The lowest two eigenmodes involve global buckling (Figure 8a, Figure 9 top): followed by axi-symmetric local buckling (Tables 1 and 2). 


\begin{tabular}{ccc}
\hline $\begin{array}{c}\text { Eigen } \\
\text { modes }\end{array}$ & \multicolumn{1}{c}{$A / L^{2}=0.01, R / t=3$} \\
\cline { 2 - 3 } 1 & mode & $\sigma_{b k} / E$ \\
2 & $\begin{array}{c}\text { global buckling } \\
\text { global buckling } \\
3\end{array}$ & 0.071 \\
4 & $\begin{array}{c}\text { non-axisymmetric } \\
\text { local buckling } \\
\text { non-axisymmetric } \\
\text { local buckling } \\
\text { axisymmetric local } \\
\text { buckling } \\
\text { axisymmetric local } \\
\text { buckling }\end{array}$ & 0.109 \\
\hline
\end{tabular}

Table 1a. Eigenvalues for $\theta=90^{\circ}$ hollow trusses

\begin{tabular}{ccc}
\hline \multirow{2}{*}{$\begin{array}{c}\text { Eigen } \\
\text { modes }\end{array}$} & \multicolumn{2}{c}{$A / L^{2}=0.05, R / t=5$} \\
\cline { 2 - 3 } 2 & mode & $\sigma_{b k} / E$ \\
3 & $\begin{array}{c}\text { non-axisymmetric } \\
\text { local buckling } \\
\text { non-axisymmetric } \\
\text { local buckling } \\
\text { axisymmetric local } \\
\text { buckling }\end{array}$ & 0.092 \\
5 & $\begin{array}{c}\text { non-axisymmetric } \\
\text { local buckling } \\
\text { axisymmetric local } \\
\text { buckling } \\
\text { non-axisymmetric } \\
\text { local buckling }\end{array}$ & 0.104 \\
6 & 0.135 \\
\hline
\end{tabular}

Table $1 b$. Eigenvalues for $\theta=90^{\circ}$ hollow trusses 


\begin{tabular}{|c|c|c|c|c|}
\hline \multirow{2}{*}{$\begin{array}{l}\text { Eigen } \\
\text { Modes }\end{array}$} & \multicolumn{2}{|c|}{$\bar{\rho}=2 \%$} & \multicolumn{2}{|c|}{$\bar{\rho}=10 \%$} \\
\hline & mode & $\sigma_{b k} / E \bar{\rho}$ & mode & $\sigma_{b k} / E \bar{\rho}$ \\
\hline 1 & global buckling & 0.023 & $\begin{array}{l}\text { non-axisymmetric } \\
\text { local buckling }\end{array}$ & 0.062 \\
\hline 2 & global buckling & 0.041 & $\begin{array}{c}\text { non-axisymmetric } \\
\text { local buckling }\end{array}$ & 0.066 \\
\hline 3 & $\begin{array}{l}\text { non-axisymmetric } \\
\text { local buckling }\end{array}$ & 0.059 & $\begin{array}{l}\text { non-axisymmetric } \\
\text { local buckling }\end{array}$ & 0.071 \\
\hline
\end{tabular}

Table 2a. Eigenvalues for the pyramidal unit cell

\begin{tabular}{ccccc}
\hline \multirow{2}{*}{$\begin{array}{c}\text { Eigen } \\
\text { Modes }\end{array}$} & \multicolumn{2}{c}{ Unit Cell } & \multicolumn{2}{c}{ Single member } \\
\cline { 2 - 5 } & mode & $\sigma_{b k} / E \bar{\rho}$ & mode & $\sigma_{b k} / E \bar{\rho}$ \\
2 & global buckling & 0.049 & global buckling & 0.049 \\
& $\begin{array}{c}\text { non- } \\
\text { axisymmetric } \\
\text { local buckling }\end{array}$ & 0.060 & non- & 0.059 \\
& $\begin{array}{c}\text { non- } \\
\text { axisymmetric }\end{array}$ & 0.063 & local buckling & non- \\
& local buckling & & axisymmetric & 0.064 \\
& & & local buckling \\
\hline
\end{tabular}

Table 2b. Comparison of Unit Cell with Single Member, $\bar{\rho}=5 \%$

Stubby trusses: characterized by $A / L^{2}=0.05, \mathrm{R} / \mathrm{t}=5$ (for $\theta=90^{0}$ ), and $\bar{\rho}=5 \%$ and $\bar{\rho}=$ $10 \%, A / L^{2}=0.0075, \mathrm{R} / \mathrm{t}=5$ (for $\theta=60^{\circ}$ ). The lowest two eigenmodes correspond to non-axi-symmetric local buckling, followed closely by axi-symmetric modes (Figure 8b, Figure 9 bottom). The lowest mode ascertained for the pyramidal cell (Figure 9) is consistent with that found upon testing a polymer micro-lattice (Figure 10). A comparison among the eigenmodes for the pyramidal truss unit cell and the single member (Figure 11) affirms that the buckling stresses and the modes are essentially the same.

Imperfections. To obtain viable predictions of plastic buckling, imperfections must be incorporated. The procedure adopted is based on that previously used for predicting the buckling of sandwich panel cores [17]. The first thirty eigenmodes are ascertained and initial imperfections are introduced to ensure that both global and local buckling effects are captured. For most of the calculations presented below, the imperfections have amplitude $1 / 10^{\text {th }}$ the shell thickness, $\xi \equiv w_{\max } / t=0.1$, where $w_{\max }$ is the maximum imperfection amplitude. 
Basis for Assessment. Extensive work on the energy absorption capabilities of cylindrical metal tubes in the post-buckling range has been reported [10-15] with application to automotive crashworthiness. The relevance of this prior work to the lattice cores is limited because, in the HRL lattice, most core members are not aligned perpendicular to the crush direction. Depending on stubbiness and $R / t$, core members display axial modes in the early stages of buckling (not unlike that experienced by a tube designed for energy absorption in normal crush) but this mode inevitably gives way to lateral buckling [16]. Design of the core members to sustain energy absorption capabilities during lateral buckling is central to the identification of effective micro-truss structures.

Truss Deformations: $\theta=90^{\circ}$. The stress/strain curves have the general form depicted on Figure 12, comprising four primary phenomena, consistent with prior assessments [16].

(i) The members yield at stress,

$\sigma / \bar{\rho} \sigma_{Y}=1$.

(ii) Strain hardening ensues followed by the onset of plastic buckling. The peak in nondimensional stress is slightly larger than unity: $\sigma_{\text {peak }} / \bar{\rho} \sigma_{Y} \approx 1.2$.

(iii) Plastic buckling is followed by softening at rate $d \sigma / d \varepsilon$ that depends sensitively on the slenderness.

(iv) For stubbier members, contact occurs at intermediate strain levels, as the tube crushes, causing a rise in the stress.

(v) Once the system begins to densify, an effective densification strain, $\varepsilon_{D}$, is reached (Figure 3b).

Calculations have been conducted for slenderness in the range, $0.0015 \leq A / L^{2} \leq 0.01$, wall thickness, $3 \leq R / t \leq 10$, and imperfection amplitudes $0 \leq \xi \leq 0.2$. A basic set of results is presented on Figures 13 and 14. The effects of the wall thickness, R/t, and of the imperfection amplitude are quite small over the range assessed. Conversely, slenderness substantially affects the response. The differing effects are evident in the deformations, in conjunction with the associated stress/strain curves. When the members are relatively stubby (Figure 13), a short wavelength shell mode is induced. The ensuing wrinkles have a characteristic wavelength smaller than the tube diameter, so that several occur over the length. Absent imperfections, axi-symmetric modes prevail (Figures 13a and $13 \mathrm{~b}$ ), but become non-axi-symmetric when imperfections are introduced (Figures 13c and 13d). These buckles develop by circumferential plastic stretching, dissipating substantial energy, and resulting in low softening rates. The softening is somewhat imperfection dependent because the non-axi-symmeteric modes reduce the requirement for plastic stretching, increasing the softening rate. The more slender tubes experience global buckling over the range of wall thickness and imperfections examined (Figure 14). The associated large-scale bending leads to rapid softening. The stresses rise again only when contact begins, at strains of order $70 \%$. 
The forgoing stress/strain curves have been converted into estimates of the energy absorbed per unit mass, by ascertaining the areas under the curves, in the manner depicted on Figure 3. The results are superimposed on Figure 5. The energies are essentially invariant with the transmitted stress, for the reason anticipated by (7b): namely, for these lattices, the peak stress sales linearly with the relative density. Note that, in the absence of softening after plastic buckling, (7b) and (13) give the upper bound:

$\Lambda \approx \varepsilon_{D} \approx 0.8$

Truss Deformations: $\theta=60^{\circ}$. The general stress/strain response is similar to that for the slender members at $\theta=90^{\circ}$. The principal difference is that the initial peak occurs at a lower axial stress, $\sigma_{\text {peak }} / \bar{\rho} \sigma_{Y} \approx 0.75$, with minimal strain hardening (Figure 15). The subsequent softening is quite extensive, and a strong function of the relative density (Figure 15), as well as the wall thickness. The deformation patterns at low relative density ( $\bar{\rho}=2 \%$ ), for imperfection amplitude (ratio of amplitude to shell thickness), $\xi=0.1$, reveal the factors that govern the response (Figure 16). Initially, the columns yield uniformly. Then, at strains of $\sim 5 \%$, just beyond the stress peak, the members buckle in accordance with eigenmode 1 (Figure 9). Upon further straining, lateral buckling occurs and kinks form at the region of highest curvature, resulting in rapid softening. In some cases, at strains of order $50-60 \%$, the kinked regions make contact causing a small elevation in the stress. The softening rate diminishes appreciably as the relative density of the tubes increases (Figure 15), because the mode changes from global buckling at low $\bar{\rho}$ to local buckling at higher $\bar{\rho}$ (Figure 9). The energy absorbed per mass is plotted on Figure 5 as a function of the relative density. Now, unlike the $90^{\circ}$ response, the energy absorbed becomes $\bar{\rho}$ dependent, because of the associated change in buckling mode.

Rate Effects: To assess the influence of inertial stabilization [17], the foregoing calculations have been repeated for compaction velocities of $100 \mathrm{~m} / \mathrm{s}$. Results obtained for slender $90^{\circ}$ members highlight the primary effect (Figure 17). Namely, global buckling is suppressed and replaced by wrinkling. The consequent energy absorption is thereby increased, as plotted on Figure 5. There is a caveat regarding the overall energy absorption in a multilayer lattice. That is, the energy elevation is a part of the extra dissipation at the shock front (Figure 4, Appendix) and should not be viewed as an additional contribution. Further evaluation of these dynamic phenomena will be addressed in continuing assessments.

\section{Experimental Methods and Measurements}

Polymer micro-lattice template fabrication: Basic polymer micro-lattices were fabricated from an interconnected pattern of self-propagating photopolymer waveguides [7-9]. A mask with a square pattern of circular apertures was placed over the photo-monomer and exposed to four collimated beams, generated from a mercury arc lamp. Each collimated 
beam had a $40^{\circ}$ incident angle, rotated $90^{\circ}$ about the normal. A pyramidal unit cell was formed by choosing a depth of photomonomer coincident with the first nodal point.

Hollow Ni micro-lattice fabrication: The polymer micro-lattices were used as direct templates for electro-deposition of Ni. Initially, a conductive seed layer $(\sim 200 \AA \mathrm{Ti}$ followed by $\sim 2000 \AA \mathrm{Au}$ ) was deposited by using an evaporator. The Ni was then electrodeposited within a commercial electroforming solution. A platinum/rhodium wire, threaded on the outer edge, formed the electrode connection. The plating was performed at $50^{\circ} \mathrm{C}$ and $\sim 8.5 \mathrm{~mA} / \mathrm{cm}^{2}$. To achieve different coating thicknesses, two different samples were plated for 6 and 12h, respectively. After electro-deposition, the top and bottom surfaces were removed to expose the underlying polymer at each node, as well as along the outer edges in contact with the electrode wire. The polymer was chemically etched in a base solution ( $3 \mathrm{M} \mathrm{NaOH}$ at $\left.60^{\circ} \mathrm{C}\right)$, creating the hollow tube Ni micro-lattice samples used for the mechanical measurements.

Base properties of electroformed $\mathrm{Ni}$ : The Ni used for fabricating the lattices had a density of 8.9g/cc. Nano-hardness tests conducted on the walls gave a Young's modulus, $\mathrm{E}=200 \mathrm{GPa}$. Micro-hardness measurements gave an inferred yield strength, $\sigma_{Y} \approx 300 \mathrm{MPa}$.

Compression Measurements. Based on their mass and dimensions, samples plated for 6 and $12 \mathrm{~h}$ had relative density, $\bar{\rho}=0.007$ and $\bar{\rho}=0.014$, respectively. The top and bottom surfaces were bonded to thick steel face-sheets using a fast-setting epoxy to constrain the nodes from lateral displacement. Quasi-static compression tests were conducted using an MTS hydraulic load frame at a strain rate of $7 \times 10^{-4} \mathrm{~s}^{-1}$. The compressive stress-strain responses for the two hollow Ni micro-lattice samples are shown in Figure 18, indicating behavior similar to that presented on Figure 3b: comprising an initial stress peak, followed by stress oscillations prior to densification at $\varepsilon_{D} \approx 0.8$. Based in the preceding estimate of the yield strength and of the relative densities, the stress peak occurs at, $\sigma_{\text {peak }} / \bar{\rho} \sigma_{Y} \approx 1.2$, slightly larger (by 20 to 30\%) than that predicted by the simulations. More significantly, the softening rate beyond the peak is much less severe than in the simulations, at the equivalent relative density. The consequence is that the energy absorbed/mass, $\Lambda \approx 1$ (beyond the scale of Figure 5 ), is about a factor 2 larger than that determined by simulation at the equivalent transmitted stress, $\sigma_{t r} / \sigma_{Y} \approx 10^{-2}(\Lambda \approx 0.4)$. The implication is that the actual lattices have plastic dissipation mechanisms not elucidated in the simulations. To search for possibilities, partially compressed lattices have been imaged (Figure 19). The images reveal that, while some members exhibit lateral buckling modes similar to predictions, a complementary local mode develops at some of the nodes. The associated local wrinkling is similar to the concertina mode identified in an earlier study [16] but remains to be understood. It is tentatively attributed to a larger R/t at the nodes. Given the implications for much greater energy absorption, this mode will be emphasized future assessments. 


\section{Concluding remarks}

Through a combination of simulations and experiments a metallic hollow wall microlattice has been identified that absorbs an unusually large energy per unit mass. The energy absorption is governed by the plastic deformations that occur subsequent to plastic buckling. In the simulations, conducted with walls of uniform thickness, the lattice members exhibit lateral buckling and kinking, with ensuing (relatively) rapid softening. Nevertheless, relative to other cellular media having isotropic response, such as foams, the hollow wall micro-lattices absorb more than twice the energy per unit mass. More dramatically, experimental measurements performed on such lattices exhibit yet larger energy absorption than the simulated lattices, by another factor of two (more than 4 to 5 times that for foams). Preliminary observations performed on the compressed lattices indicate a local wrinkling mode in the vicinity of the nodes where $\mathrm{R} / \mathrm{t}$ is larger. The associated concertina mode is surmised to be the source of the extra energy absorption. This exciting new development is the subject of ongoing research.

\section{Appendix: Analysis of shock effects and design metrics}

We seek to determine the minimum thickness $h=h_{\min }$ of a multilayer cellular medium needed to dissipate the kinetic energy $(1 / 2) m_{b} v_{0}^{2}$ of the buffer. The medium has initial density $\rho$ and the compressive stress/strain characteristic (under uniaxial straining) as sketched in Fig. A1a: with a constant plateau stress $\sigma_{p l}$ and a nominal densification strain $\varepsilon_{D}$. A plastic shock enters the medium from the impact face and travels at a speed $c(t)$. The incident portion, downstream of the shock, attains a velocity $v(t)$ (Fig. A1b). The distal portion, upstream, is at rest. At any instant, the medium is non-deforming except for a jump in compressive strain (magnitude $\varepsilon_{D}$ ) across the shock. After time $t$, the shock has traveled distance $s$, as measured in the un-deformed configuration. We proceed to analyze the shock employing a Lagrangian framework wherein all velocities and accelerations are measured with respect to a stationary reference frame. For this one-dimensional situation (with zero plastic Poisson ratio), the Cauchy and nominal stresses are identical and the equation of motion is:

$$
\frac{\partial \sigma}{\partial X}=-\rho \frac{\partial^{2} u}{\partial t^{2}}
$$

where $X$ is the position of material point in the un-deformed configuration, $u$ is displacement, and $\sigma$ the stress. Integrating (A. 1) over the range $0 \leq X \leq s$ and noting that the medium (and buffer) are rigid, gives the acceleration $\dot{v}$ downstream of the shock as:

$$
\left(m_{b}+\rho s\right) \dot{v}=-\sigma_{d}
$$

Immediately upstream the stress is $\sigma_{p l}$. Momentum conservation across the shock dictates that [10] 


$$
\sigma_{d}-\sigma_{p l}=\rho c v
$$

Furthermore, mass conservation implies that [10]

$$
\dot{s} \equiv C=v / \varepsilon_{D}
$$

Combining (A. 2) to (A. 4) gives the overall governing ODE for the deformation as

$$
\frac{m_{b}}{\rho} \ddot{s}+s \ddot{s}+\dot{s}^{2}=-\frac{\sigma_{p l}}{\rho \varepsilon_{D}} .
$$

The initial conditions are $s=0$ and $\dot{s}=v_{0} / \varepsilon_{D}$ at time $t=0$. When the deformation arrests, at time, $t=M / \sigma_{p l}$, then $s=s_{\text {final }}$ and we define $h_{\min } \equiv s_{\text {final }}$.

The non-dimensional groups are

$$
\bar{s} \equiv \frac{s}{h} ; \quad \bar{t} \equiv \frac{t v_{0}}{h} ; \quad \dot{\bar{s}} \equiv \frac{\dot{s}}{v_{0}} ; \ddot{\bar{s}} \equiv \frac{\ddot{s} h}{v_{0}^{2}}
$$

Substituting into (A. 5), the governing ODE becomes

$$
\ddot{s} / \Pi+\bar{s} \ddot{\bar{S}}+\dot{\bar{s}}^{2}=-\Psi / \varepsilon_{D}^{2}
$$

with initial conditions, $\bar{s}=0$ and $\dot{\bar{s}}=1 / \varepsilon_{D}$ at $\bar{t}=0$. One of the associated nondimensional parameters is the mass ratio for the cellular medium to the buffer

$$
\Pi \equiv \frac{\rho h}{m_{b}}
$$

while the other is the ratio of the static to dynamic strength of the medium

$$
\begin{aligned}
& \Psi \equiv \frac{\sigma_{p l} m_{b}^{2} \varepsilon_{D}}{\rho M^{2}} \\
& =\frac{\sigma_{p l}}{\rho v_{0}^{2} / \varepsilon_{D}}
\end{aligned}
$$

The response is completely specified given the non-dimensional quantities, $\Pi, \Psi$ and $\varepsilon_{D}$. We iteratively solve (A. 7) for $\Pi_{\min }$ such that $\bar{s}=1$, when $\dot{\bar{s}}$ first attains zero. The numerical values are plotted on Fig.4 as a function of $\Psi$. Two regimes emerge.

Regime I ( $\Pi<<1)$ : wherein Eq. (A. 7) can be approximated as

$$
\frac{\ddot{\bar{S}}}{\Pi}=-\frac{\Psi}{\varepsilon_{D}^{2}}
$$

This ODE can be solved analytically (with the foregoing initial conditions) to obtain 


$$
\dot{\bar{S}}^{2}=\frac{1}{\varepsilon_{D}^{2}}-\frac{2 \Psi \Pi}{\varepsilon_{D}^{2}} \bar{s}
$$

Enforcing the condition that $\bar{s}=1$ when $\dot{\bar{s}}=0$, we obtain

$$
\Pi_{\min }=1 / 2 \Psi
$$

or, equivalently

$$
h_{\min }=\frac{M^{2}}{2 \sigma_{p l} m_{b} \varepsilon_{D}} .
$$

In this regime the buffer is much heaver than the cellular medium, whereupon inertial effects can be neglected and the quasi-static energy balance equation holds:

$$
\sigma_{p l} \varepsilon_{D} h_{\min }=(1 / 2) m_{b} v_{0}^{2}
$$

The minimum medium thickness decreases with increasing mass $m_{b}$ of the buffer because the kinetic energy it acquires varies as, $M^{2} / 2 m_{b}$.

Regime II ( $\>>1$ ): wherein Eq. (A. 7) can be approximated as

$$
\bar{S} \ddot{\bar{S}}+\dot{\bar{S}}^{2}=-\frac{\Psi}{\varepsilon_{D}^{2}}
$$

with initial conditions $\bar{s}=1 / \Pi$ and $\dot{\bar{s}}=1 / \varepsilon_{D}$ at $\bar{t}=0$. These modified initial conditions ensure that, $\ddot{\bar{s}}=-\left(\Pi / \varepsilon_{D}^{2}\right)(\Psi+1)$ at $\bar{t}=0$, consistent with Eq. (A. 7). Again, Eq. (A. 10) can be solved analytically to give

$$
\bar{s}=\frac{1}{\Pi} \sqrt{\frac{\Psi+1}{\Psi+\varepsilon_{D}^{2} \dot{\bar{S}}^{2}}}
$$

Substituting the condition that, when $\dot{\bar{s}}=0, \bar{s}=1$ we obtain

$$
\Pi_{\min }=\sqrt{1+\frac{1}{\Psi}}
$$

Recalling that this solution only holds for $\Pi>>1$, it is sufficient to approximate as

$$
\Pi_{\min } \approx \frac{1}{\sqrt{\Psi}}
$$


or equivalently

$$
h_{\min } \approx \frac{M}{\sqrt{\rho \sigma_{p l} \varepsilon_{D}}}
$$

In this regime the buffer has lower weight than the cellular medium, whereupon inertial effects are significant. The energy absorbed significantly exceeds the quasi-static dissipation. This extra dissipation can be traced to the very high strain rates across the shock that give rise to, among other things: changes in the collapse mode of the individual cells, inertial stabilization of the struts, a change in the collapse mode from bending/buckling to axial stretching of the struts.

Equations (A. 3) and (A. 4) together specify that

$$
\sigma_{d}=\sigma_{p l}+\rho \frac{v_{0}^{2}}{\varepsilon_{D}}
$$

Thus, the ratio $\Omega$ of the energy absorption under dynamic and quasi-static $\left(v_{0} \rightarrow 0\right)$ conditions is

$$
\Omega=1+\frac{\rho v_{0}^{2}}{\sigma_{p l} \varepsilon_{D}}
$$

Namely, the energy absorption increases quadratic ally with increasing $v_{0}$. A consequence is that, unlike regime $I, h_{\min }$ is independent of $m_{b}$, because the increased energy absorption compensates for the increased kinetic energy of the buffer.

The asymptotic solutions, included in Fig.4, affirm that Eqs. (A. 8c) and (A. 13a) are a good approximation for the full numerical solution in the ranges, $\Pi_{\min } \leq 2$ and $\Pi_{\min }>2$.

Optimization of the protection system. It is of interest to minimize either the mass or volume of the entire protection system comprising the cellular medium and buffer. For this purpose, the buffer mass/thickness is treated as the free variable and we employ the analytical solutions for $h_{\min }$.

Minimum mass systems. The total mass/area of the protection system is

$$
m_{\text {total }}=m_{b}+\rho h_{\min }
$$

Regime I: Substituting for $h_{\min }$ from Eq. (A. 8d) we obtain

$$
m_{\text {total }}=m_{b}+\frac{\rho M^{2}}{2 \sigma_{p l} m_{b} \varepsilon_{D}}
$$

Minimizing $m_{\text {total }}$ with respect to $m_{b}$, gives the minimum mass of the total system as

$$
m_{\text {total }}^{\min }=M \sqrt{\frac{2 \rho}{\sigma_{p l} \varepsilon_{D}}}
$$


with $\Pi_{\min }=1$ at the optimum. Recall that this regime applies when $\Pi_{\min } \leq 2$.

Regime II: Substituting for $h_{\text {min }}$ from Eq. (14b) we obtain

$$
m_{\text {total }}=m_{b}+M \sqrt{\frac{\rho}{\sigma_{p l} \varepsilon_{D}}}
$$

As $h_{\text {min }}$ is independent of $m_{b}$, there is no minimum for $m_{\text {total }}$. Recalling that Eq. (A. 13) is only valid for $\Pi_{\min }>2$, gives the restriction that $m_{b}$ satisfy

$$
m_{b}<\frac{M}{2} \sqrt{\frac{\rho}{\sigma_{p l} \varepsilon_{D}}}
$$

To ensure that the minimum mass solutions are lighter than those for regime I we restrict solutions to the range

$$
m_{b} \leq(\sqrt{2}-1) M \sqrt{\frac{\rho}{\sigma_{p l} \varepsilon_{D}}}
$$

Whereupon, the overall minimum mass solution always lies in regime II and given by Eq. (A. 19) with $m_{b}$ specified by Eq. (A. 21).

Minimum volume systems. In some applications it may be necessary to minimize the volume of the protection system. The volume/area (or equivalently, the thickness) is given by

$$
V_{\text {total }}=\frac{m_{b}}{\rho_{b}}+h_{\min }
$$

where $\rho_{b}$ is the density of the buffer material. We minimize $V_{\text {total }}$ with respect to $m_{b}$.

Regime I: Substituting for $h_{\min }$ from Eq. (10b) we obtain

$$
V_{\text {total }}=\frac{m_{b}}{\rho_{b}}+\frac{M^{2}}{2 \sigma_{p l} m_{b} \varepsilon_{D}}
$$

Minimizing $V_{\text {total }}$ with respect to $m_{b}$ gives the minimum volume as

$$
V_{\text {total }}^{\min }=M \sqrt{\frac{2}{\rho_{b} \sigma_{p l} \varepsilon_{D}}}
$$

with

$$
\Pi_{\min }=\rho / \rho_{b}
$$

Thus, the minimum volume solution is only valid for the choice, $\rho_{b} / \rho \geq 2$. This, constraint is practical, as we anticipate the density of most buffer plate materials to be more than twice that of the backing foam.

Regime II: In this regime the volume of the protection system is given by 


$$
V_{\text {total }}=\frac{m_{b}}{\rho_{b}}+M \sqrt{\frac{1}{\rho \sigma_{p l} \varepsilon_{D}}}
$$

25)

Since $h_{\min }$ is independent of $m_{b}$, there is no minimum for $V_{\text {total }}$.

The global minimum must ensure that the minimum $V_{\text {total }}$ is less than or equal to the volume specified by Eq. (A. 24a), giving the additional constraint

$$
0<m_{b} \leq M\left(\sqrt{2}-\sqrt{\frac{\rho_{b}}{\rho}}\right) \sqrt{\frac{\rho_{b}}{\sigma_{p l} \varepsilon_{D}}}
$$

Combining with Eqn (A. 20) gives the constraints on $m_{b}$ as

$$
m_{b} \leq \begin{cases}\frac{M}{2} \sqrt{\frac{\rho}{\sigma_{p l} \varepsilon_{D}}} & \text { if } \frac{\rho_{b}}{\rho} \leq \frac{1}{2} \\ M\left(\sqrt{2}-\sqrt{\frac{\rho_{b}}{\rho}}\right) \sqrt{\frac{\rho_{b}}{\sigma_{p l} \varepsilon_{D}}} & \text { if } \frac{1}{2}<\frac{\rho_{b}}{\rho} \leq 2 \\ \text { no solution } & \text { for } \quad \frac{\rho_{b}}{\rho}>2\end{cases}
$$

However, in most cases, it is impractical to obtain a material combination with $\rho_{b} \leq 2 \rho$. Accordingly, we anticipate that practical designs will lie in regime I, given by Eq. (A. 24)

Choice of material. The mass of the protection system is minimized by choosing a cellular medium that maximizes its quasi-static energy absorption/mass, $U_{m}=\left(\sigma_{p l} \varepsilon_{D}\right) / \rho$, with no constraint on the choice of the buffer material. The volume of the protection system is minimized by choosing a cellular medium that maximizes its quasi-static energy absorption/volume $\left(\sigma_{p} / \varepsilon_{D}\right)$ and choosing a dense buffer material (high $\rho_{\mathrm{b}}$ ). 


\section{References}

[1] M. F. Ashby, A. G. Evans, N. A. Fleck, L. J. Gibson, J. W. Hutchinson, H. N. G. Wadley, Metal Foams, A Design Guide, Butterworth-Heinemann, 2000.

[2] H. N. G. Wadley, K.P. Dharmasena, M. Y. He, R. M. McMeeking, A. G. Evans, T. BuiTanh and R. Radovitzky, Intl. J. Impact Engineering (2009) in press

[3] L. J Gibson, M. F. Ashby, Cellular solids: Structures and Properties, Cambridge University Press, 1988.

[4] A. G. Evans, J. W. Hutchinson, N. A. Fleck, M. F. Ashby, H. N. G. Wadley, Progress in Materials Science, 46 (2001) 309-327.

[5] G. W. Kooistra, V. S. Deshpande, H. N. G. Wadley, Acta Materialia, 52 (2004) 4229-

4237

[6] D. T. Queheillalt, H. N. G. Wadley, Acta Materialia, 53 (2005) 303-313.

[7] A. J. Jacobsen, W. B. Carter, S. Nutt, Advanced Materials, 19 (2007) 3892-3896.

[8] A. J. Jacobsen, W. B. Carter, S. Nutt, Acta Materialia, 55 (2007) 6724-6733.

[9] A. J. Jacobsen, W. B. Carter, S. Nutt, (2007). Acta Materialia, 56 (2007) 2540-2548.

[10] P. J. Tan, S. R. Reid, J. J. Harrigan, Z. Zou, S. Li, Journal of the Mechanics and Physics of Solids, 53 (2005) 2206-2230

[11] N. Jones. Structural Impact, Cambridge University Press, Cambridge, UK, 1989.

[12] Abramowicz, W., Jones, N., International Journal of Impact Engineering, 19 (1997) 415-

437.

[13] J. M. Alexander. Quarterly Journal of Mechanics and Applied Mathematics, 13 (1960) 10-15.

[14] T. Wierzbicki, S. U. Bhat, W. Abramowicz, International Journal of Solids and Structures, 29 (1992) 3269-3288.

[15] T. Wierzbicki, W. Abramowicz Journal of Applied Mechanics, 50 (1983) 727-734.

[16] S. M. Pingle, N. A. Fleck, V. S. Deshpande and H. N. G. Wadley, to be published

[17] E. Ferri, E. Antinucci, M. Y. He, J. W. Hutchinson, F. W. Zok Evans A.G. Journal of Mechanics of Materials and Structures, 1 (2006) 1345-1365.

[18] S. S. Hsu, N. Jones, International Journal of Crashworthiness, 9 (2004) 195-217. 
(a)
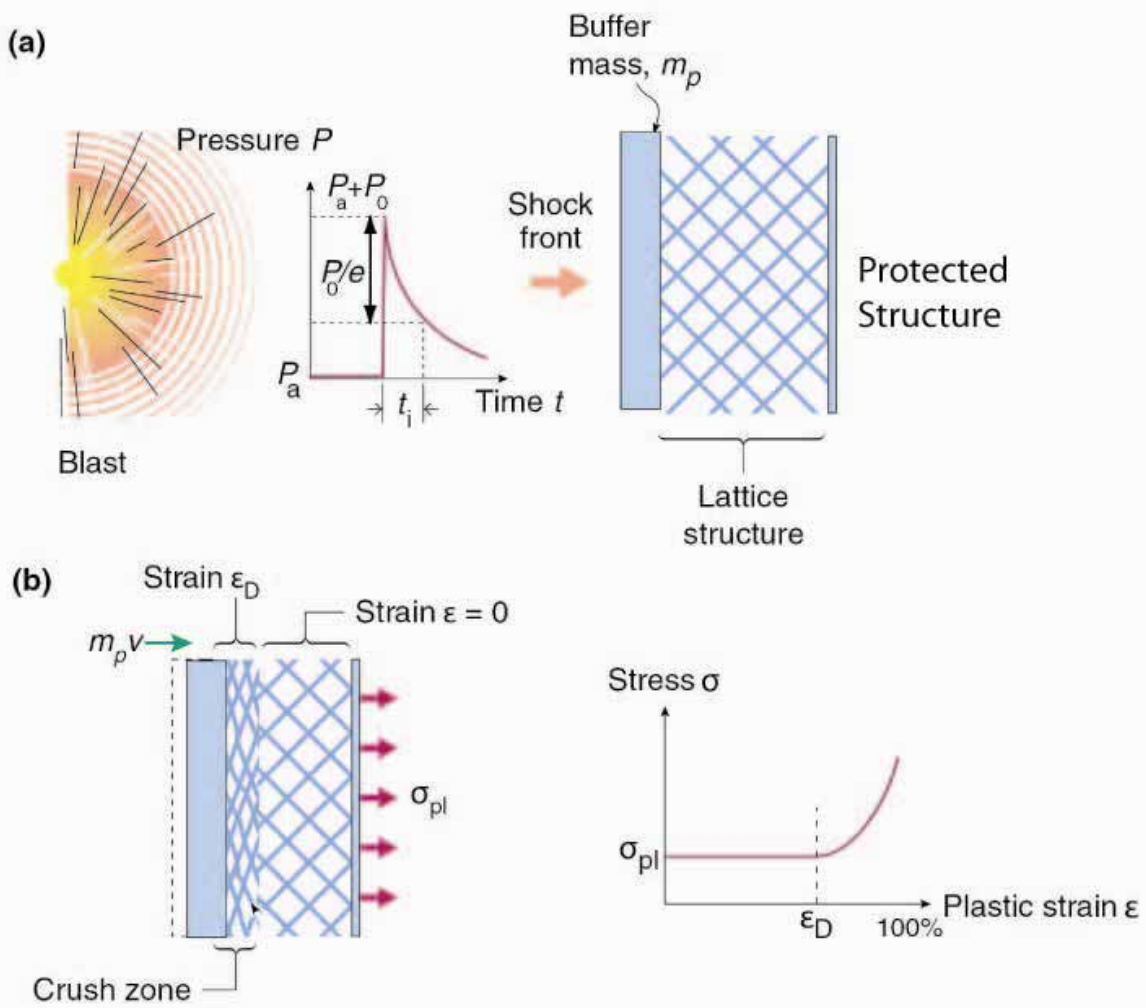

Figure 1. A schematic showing the impulse from an air blast impinging on a cellular medium attached to a solid buffer used for rectification. Also shown is the compression of the cellular medium in response to the KE imparted to the buffer and the simplified form of the stress/strain response[2].
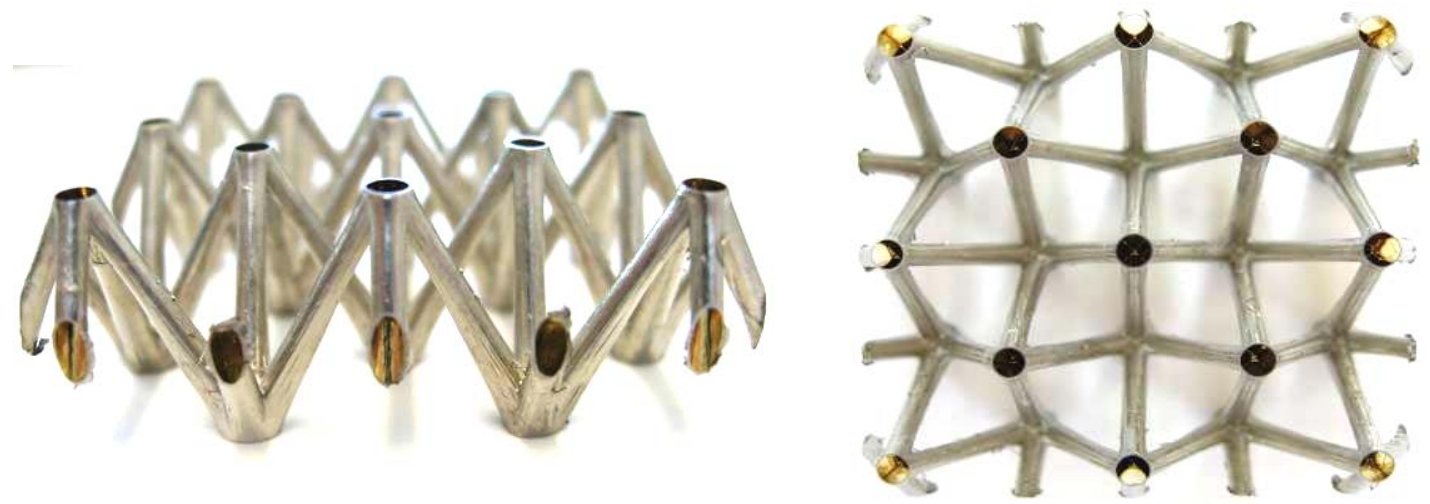

Figure 2 (a). An image of an HRL micro-lattice. (b) An image of a hollow truss Ni microlattice. 

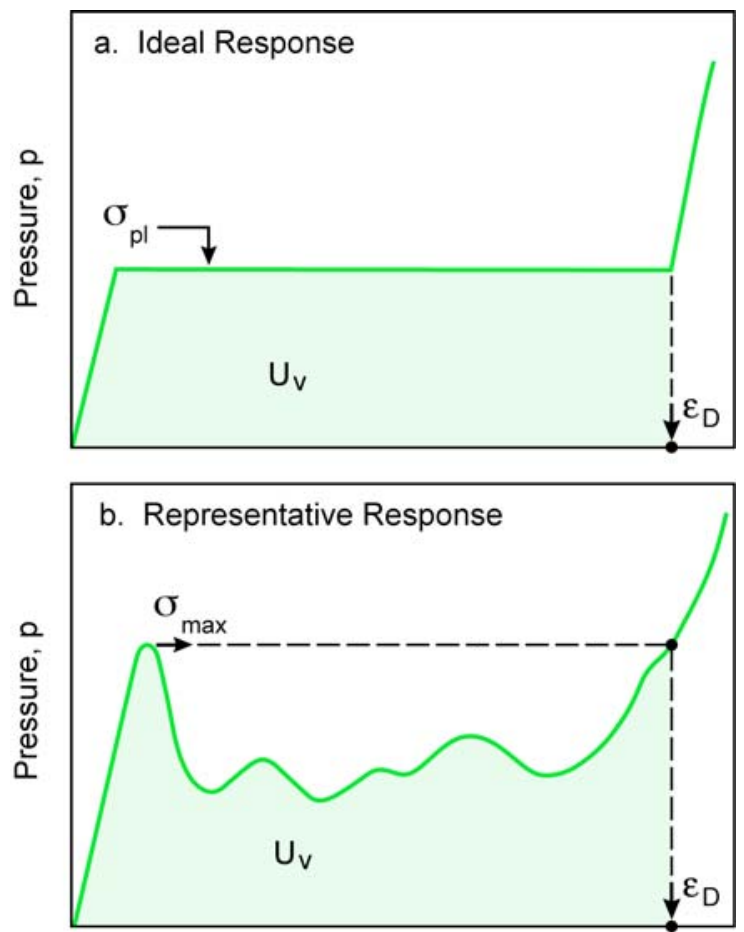

Compressive Strain, $\varepsilon$

Figure 3. Response of cellular solids to compression. (Top) "Ideal” behavior found in some foams. (Bottom) Actual behavior found in lattice solids and prismatic systems.

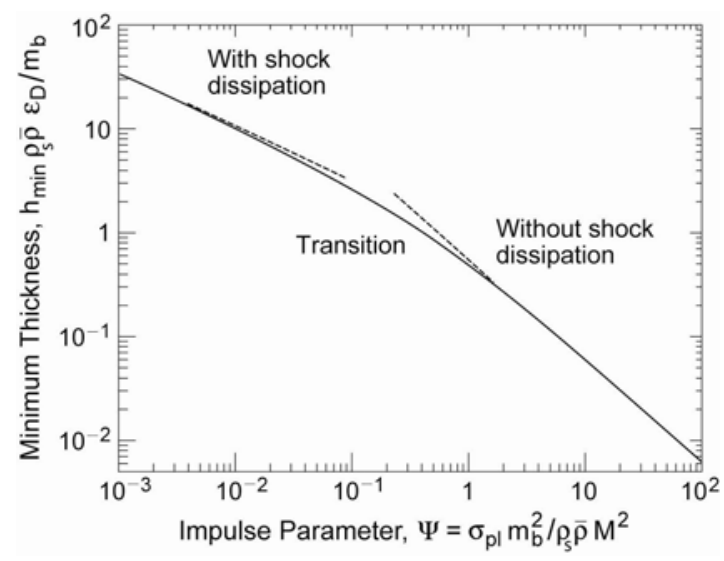

Figure 4. The minimum thickness of a cellular medium needed to absorb the KE from an impulse. 

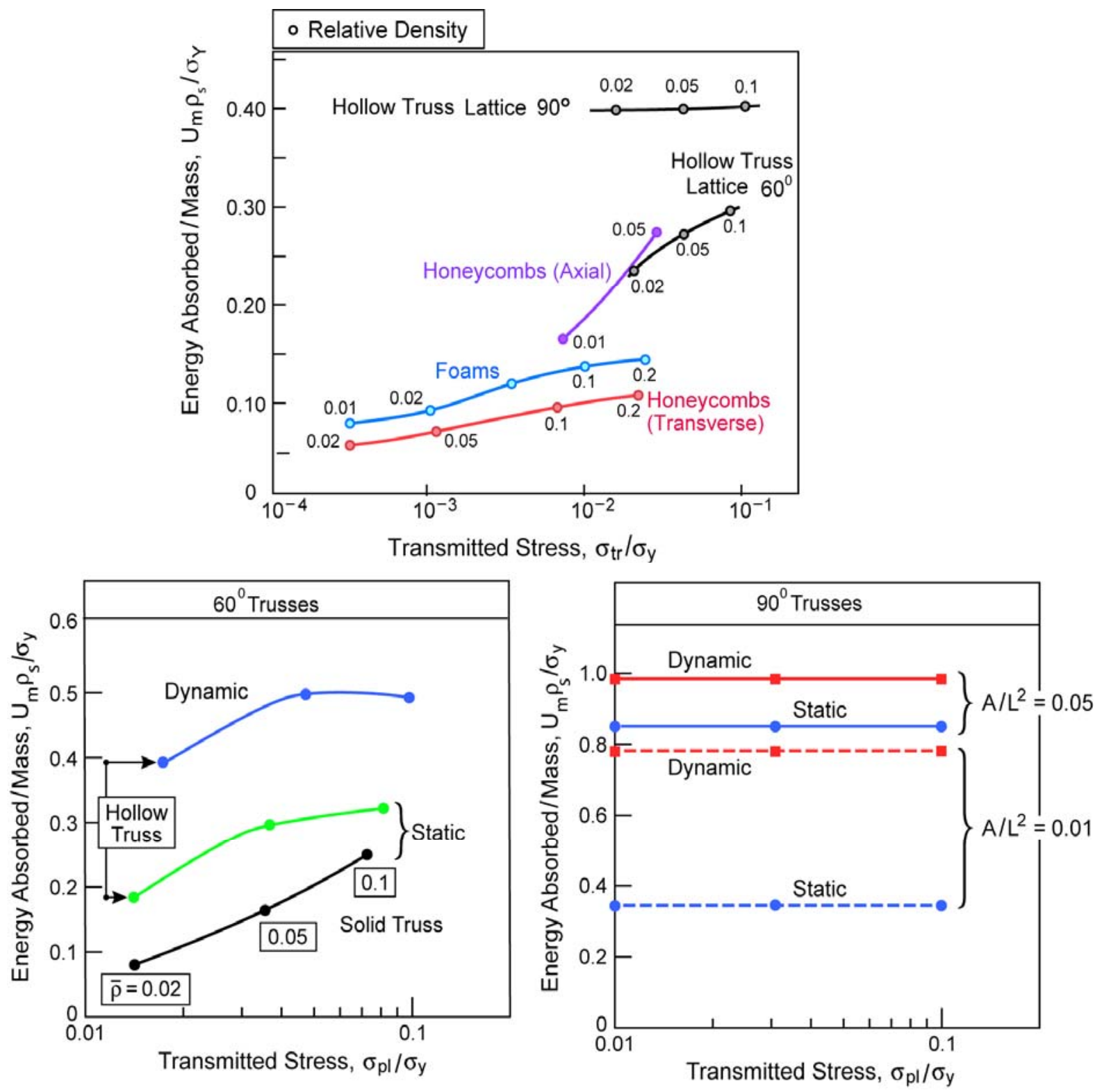

Figure 5. Relationships between the energy absorbed/mass as a function of the stress transmitted through the medium: presented in a non-dimensional form that distinguishes the effects of topology from the influence of material properties. (Top) Comparison between hollow truss microlattices and conventional cellular media. (Bottom). Results for a range of solid and hollow truss lattices: both quasi-static and dynamic. For all the hollow truss, $R / t=5$. 


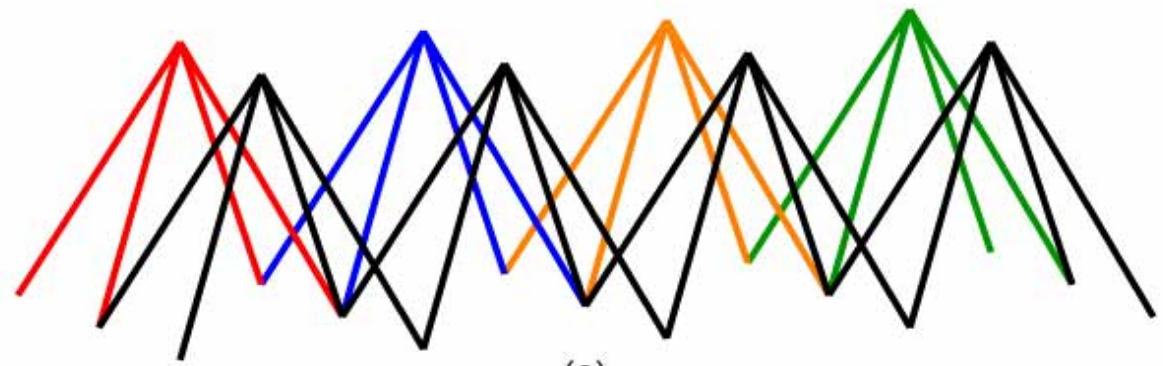

(a)

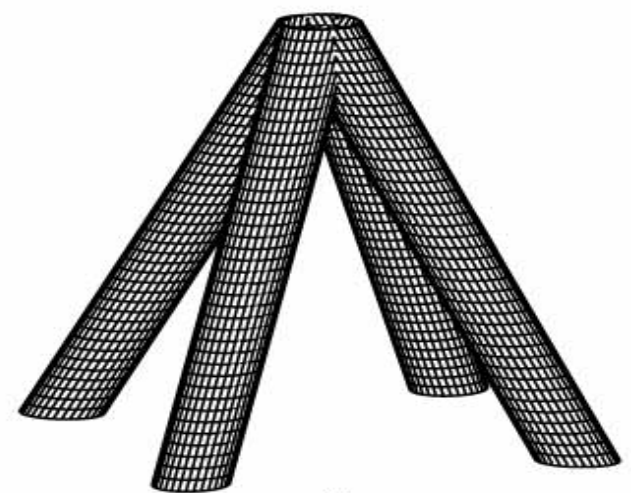

(b)

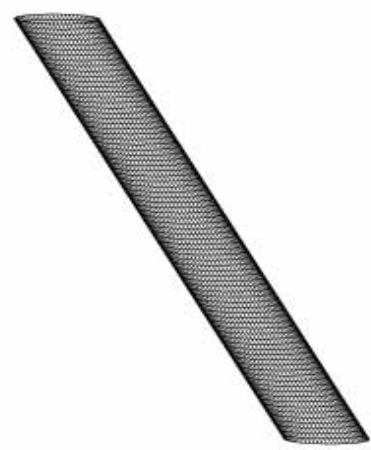

(c)

Figure 6. Finite element schemes: (a) beam elements for 4 by 2 cells, (b) 3D shell elements, pyramidal truss unit cell, (c) 3D shell elements, single member 1/4 cell.

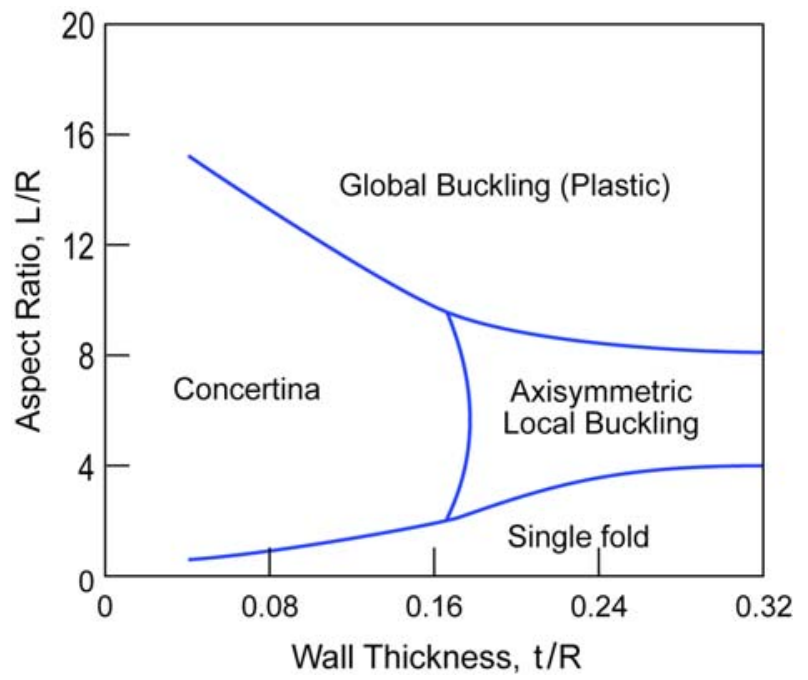

Figure 7. Failure mechanism map for hollow $90^{\circ}$ lattice members tested in compression [16]. 


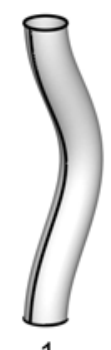

1

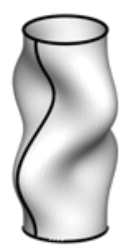

1

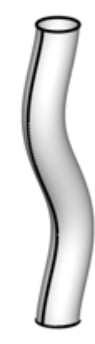

2

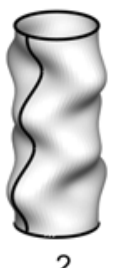

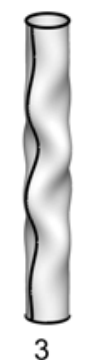

(a)

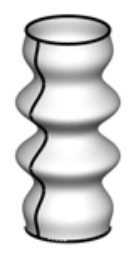

3

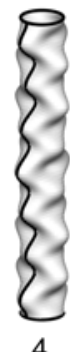

4

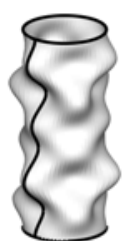

4
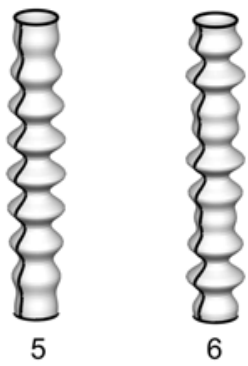

(b)

Figure 8 The six Eigenmodes with lowest buckling stress for the $90^{\circ}$ hollow tube. The stresses are given in table I. (Top) Slender members, $A / L^{2}=1 \%, R / t=3$ : The two lowest modes refer to global buckling. The other four are for local buckling in axi-symmetric and non-axisymmetric modes. (Bottom) Stubby members $A / L^{2}=5 \% R / t=5$ : all modes involve local buckling.
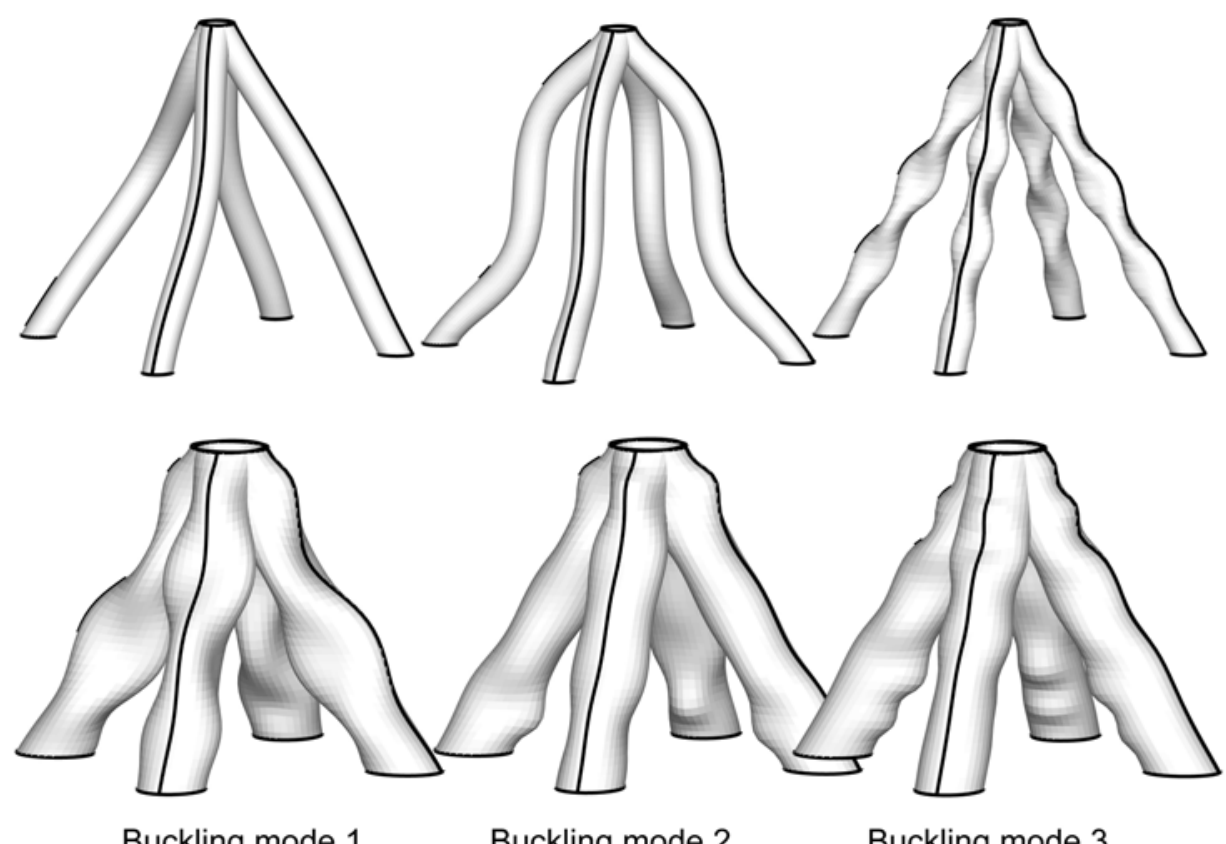

Figure 9. Three lowest Eigenmodes for the pyramidal truss unit cell with $R / t=5$. The corresponding buckling stresses are summarized on table 2. (Top) Low relative density, $\bar{\rho}=2 \%$. The lowest two coincide with global buckling, while the third involves nonaxisymmetric local buckling. (Bottom) Higher relative density, $\bar{\rho}=10 \%$ : all three modes are local. 


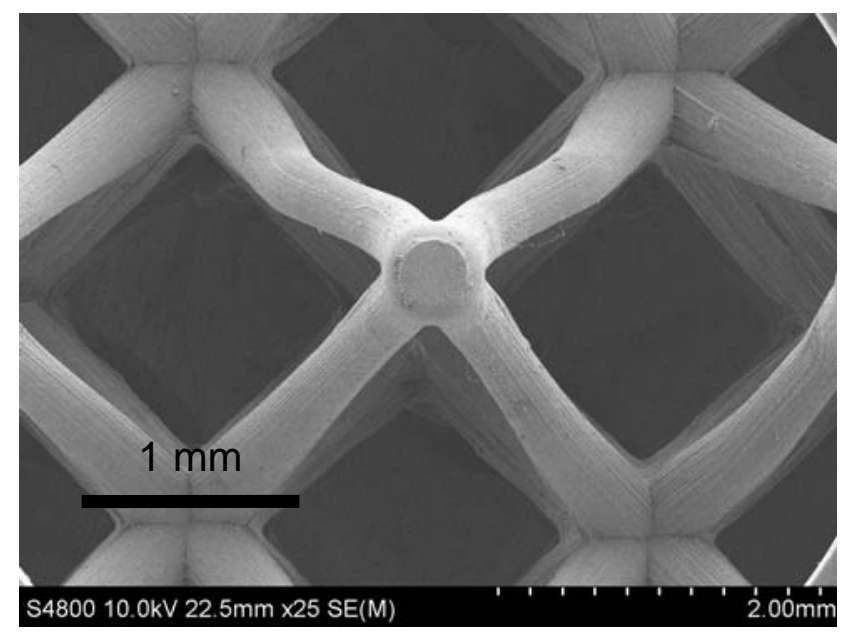

Figure 10. The buckling mode for a polymer micro-lattice, showing the correspondence with the first mode depicted on figures 9 and 11.

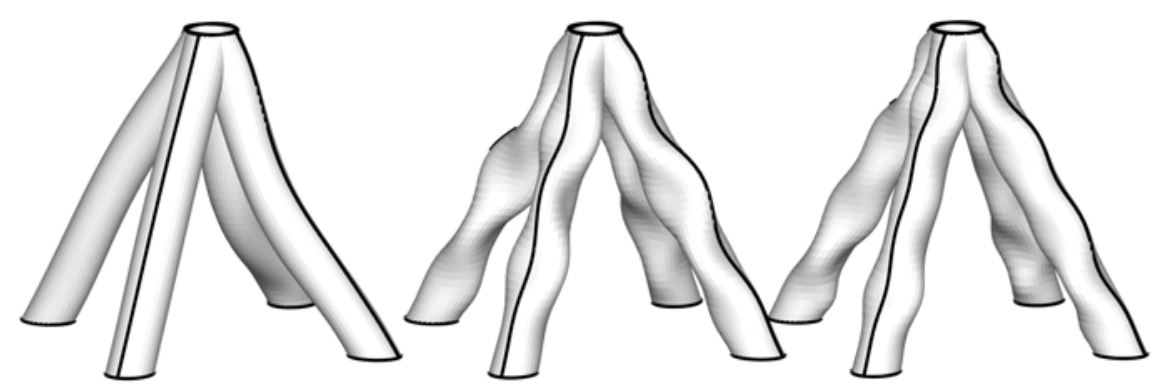

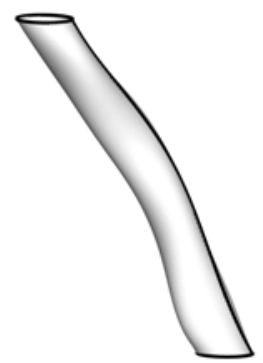

Buckling mode 1

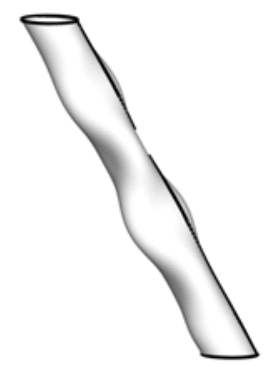

Buckling mode 2

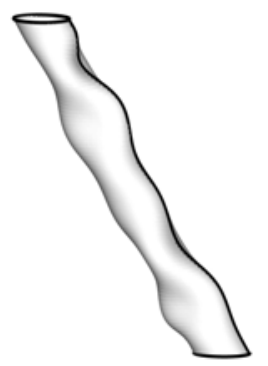

Buckling mode 3

Figure 11. The close correspondence between the Eigenmodes for the pyramidal truss unit cell (top) and a single member subject to symmetry boundary conditions (bottom): relative density $\bar{\rho}=5 \%, R / t=5$. 


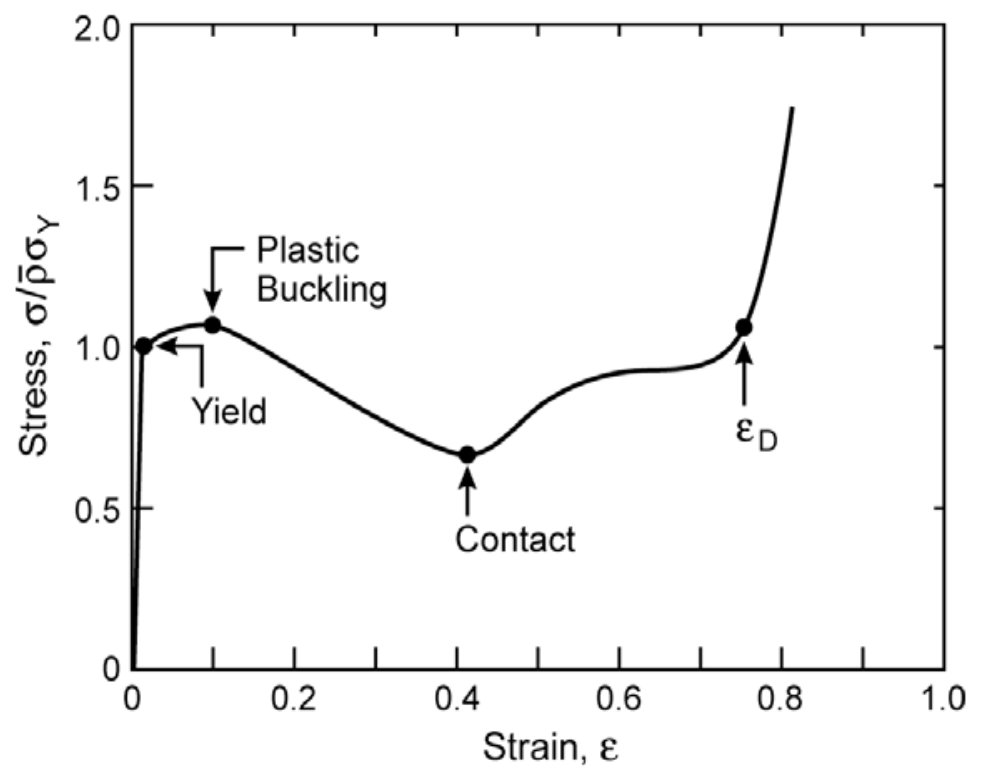

Figure 12. A representative stress/strain curve found for hollow truss micro-lattices with $90^{\circ}$ truss orientation. 

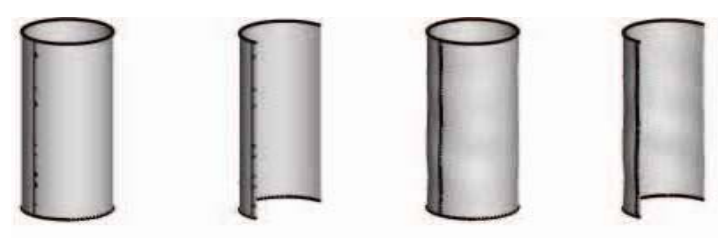

$$
\text { A: } \varepsilon=0
$$
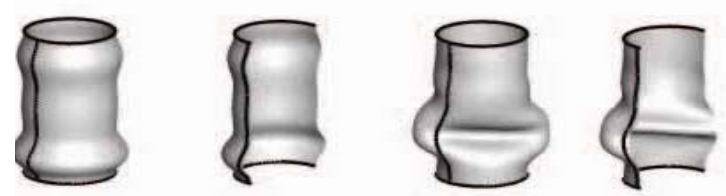

B: $\varepsilon=20 \%$
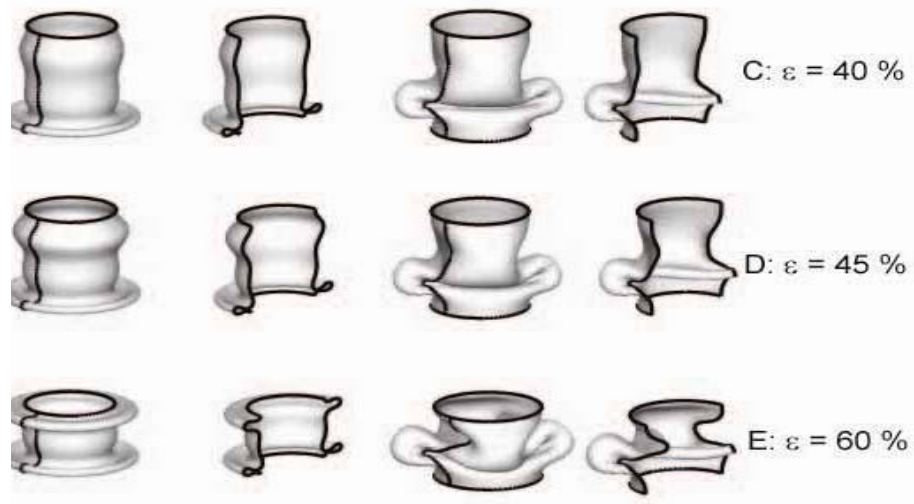

(a)

(b)

(c)

(d)

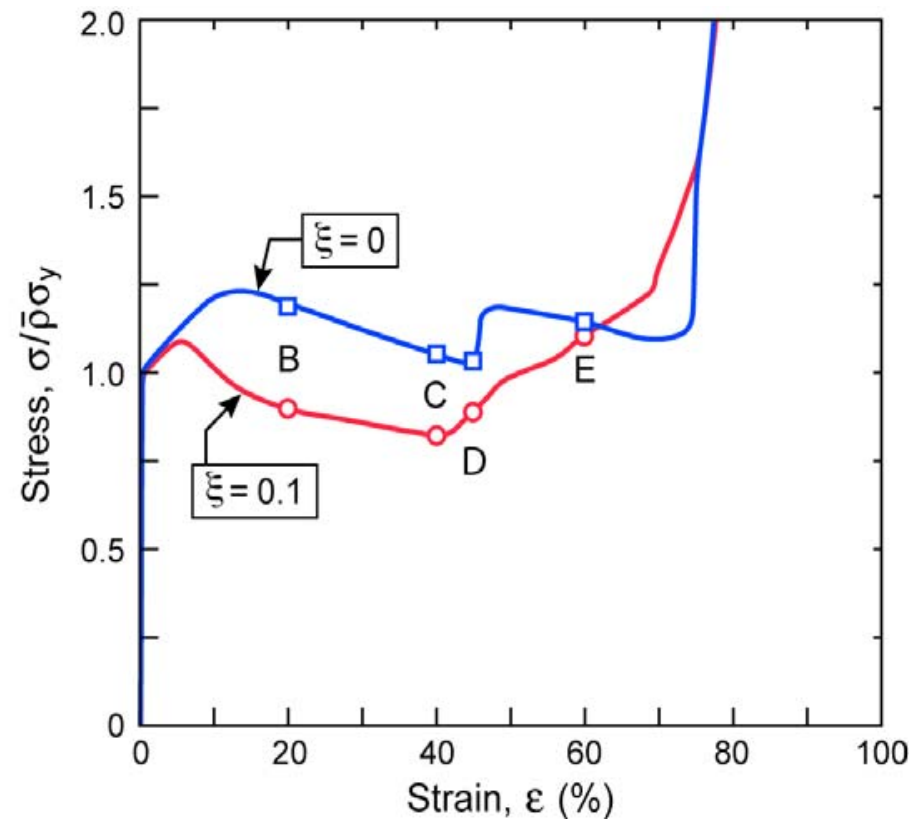

Figure 13. Stress/strain response and deformation characteristics for a stubby $90^{\circ}$ hollow tube, comparing a case without imperfections, $\xi=0$ (upper left columns, $a, b$ ) to a case incorporating imperfections (upper right columns, $c$, d) with amplitude, $\xi=0.1$ (described in the text): $R / t=5, A / L^{2}=5 \%$. The letters on the lower plots refer to the deformation responses shown on the upper figure. Note the circumferential plastic stretch at the location of the local buckles. Columns ( $a$ and $c$ ) show the deformations of the full tube. Columns ( $b$ and $d$ ) show mid-plane sections through the tube that reveal additional detail. 

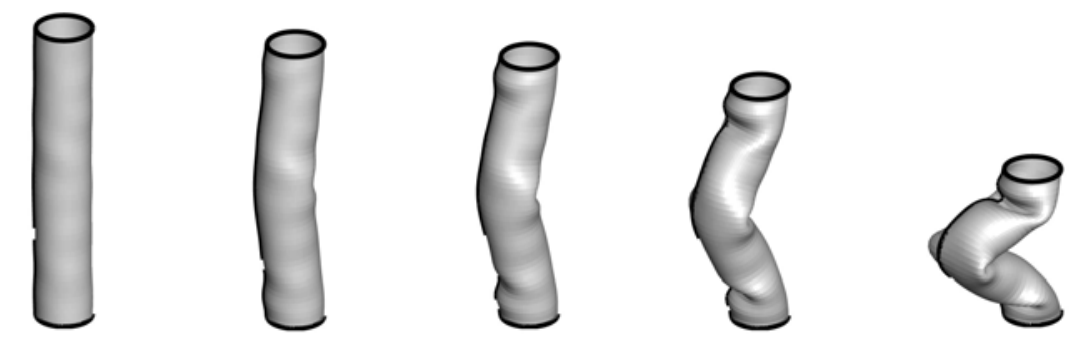
$\varepsilon=0$
$A: \varepsilon=5 \%$
B: $\varepsilon=10 \%$
C: $\varepsilon=20 \% \quad$ D: $\varepsilon=50 \%$

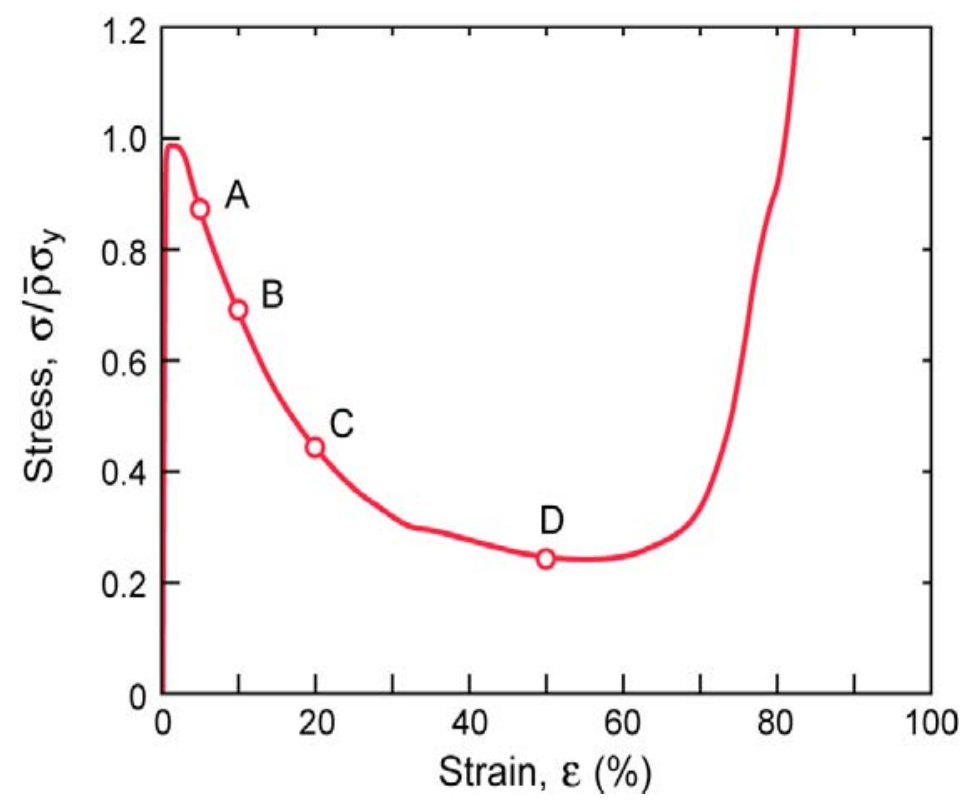

Figure 14. Stress/strain response and deformation characteristics for a slender $90^{\circ}$ hollow tube for a case incorporating imperfections with amplitude, $\xi=0.1$ (described in the text): $R / t=5, A / L^{2}=1 \%$ 


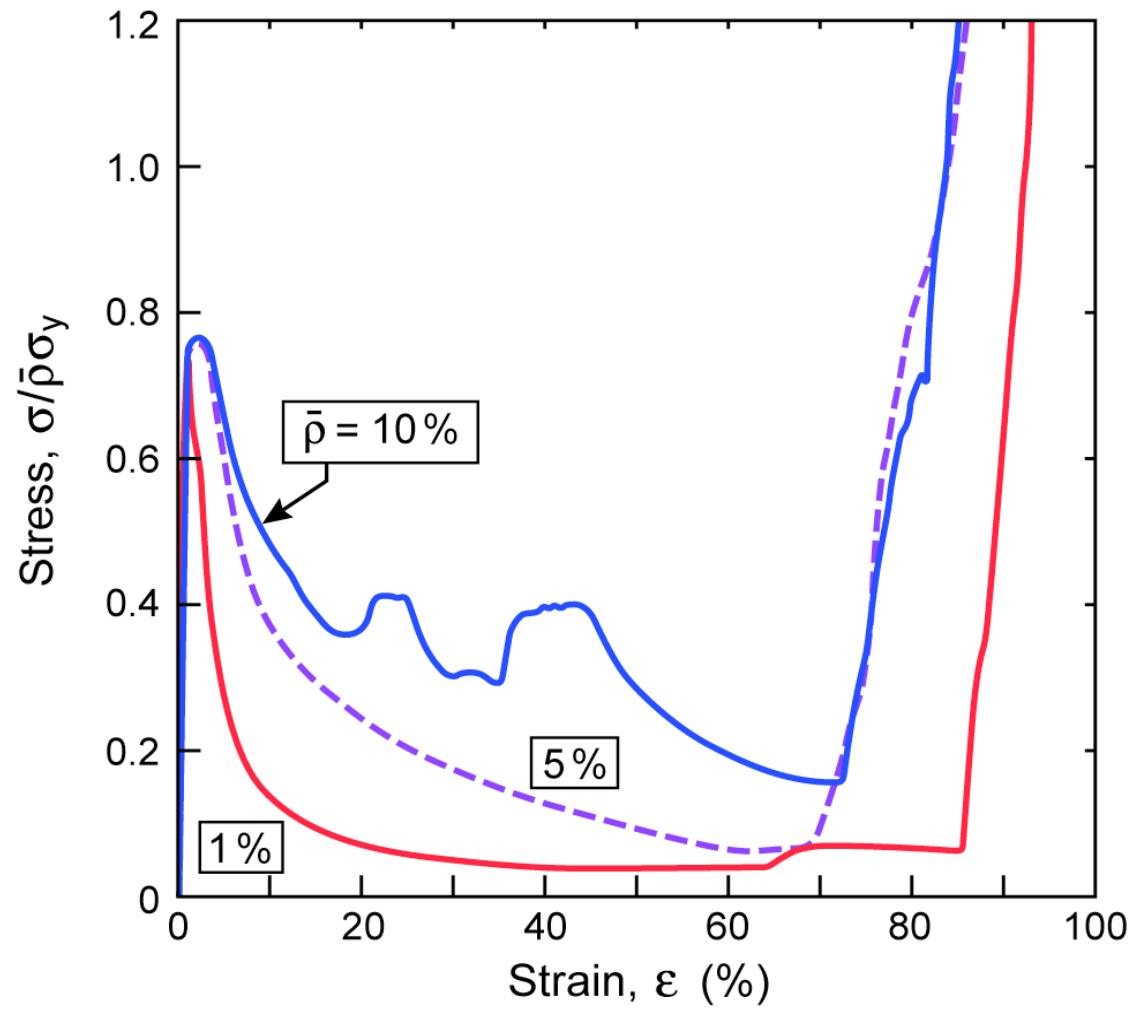

Figure 15. Effects of relative density on the stress/strain response of a $60^{\circ}$ pyramidal hollow truss lattice: $R / t=10$. 


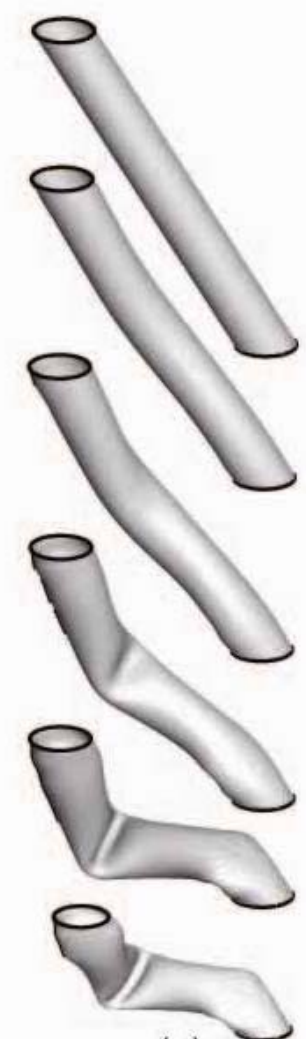

(a)

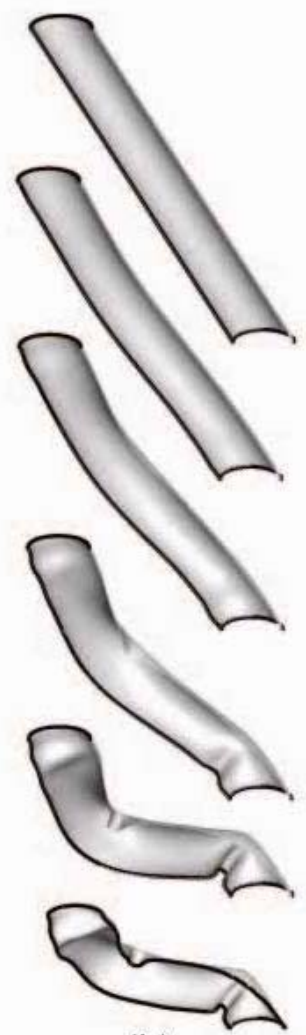

(b)

A: $\varepsilon=0$

B: $\varepsilon=5 \%$

C: $\varepsilon=10 \%$

$D: \varepsilon=20 \%$

$\mathrm{E}: \varepsilon=50 \%$

$\mathrm{F}: \varepsilon=60 \%$

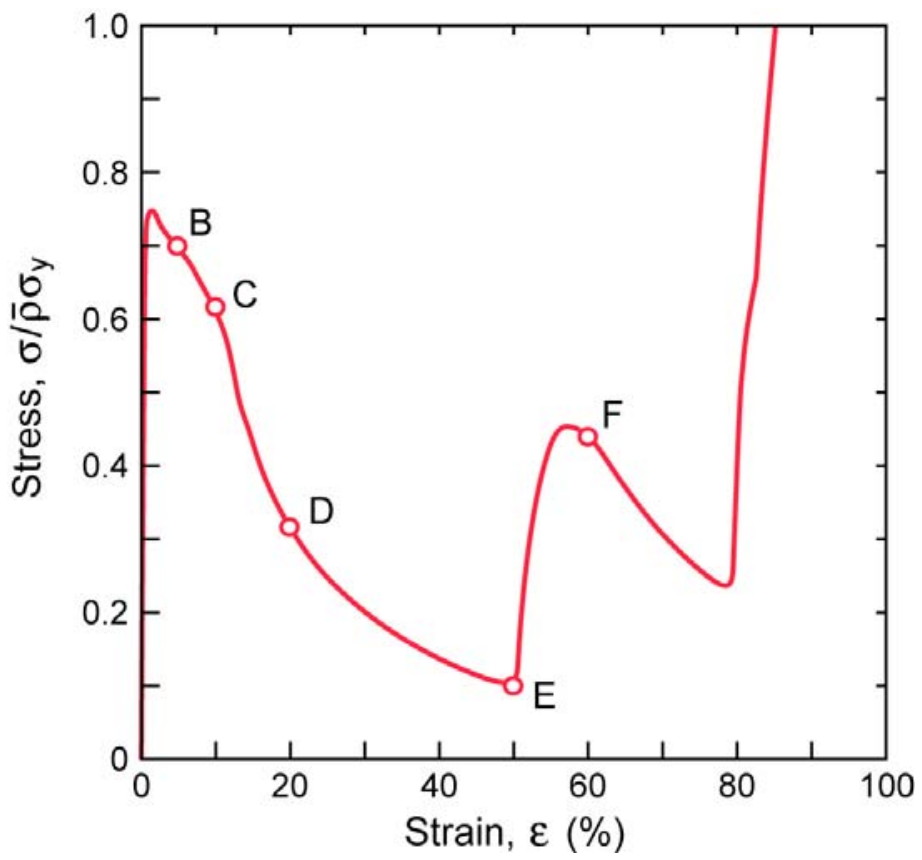

Figure 16. Stress/strain response and deformation characteristics for a slender $60^{\circ}$ hollow micro-lattice for a case incorporating imperfections with amplitude, $\xi=0.1$ : $R / t=5, \bar{\rho}=5 \%$. The upper left figures (a) show the deformations of the full tube. The upper right figures (b) show mid-plane sections through the tube that reveal additional detail. 


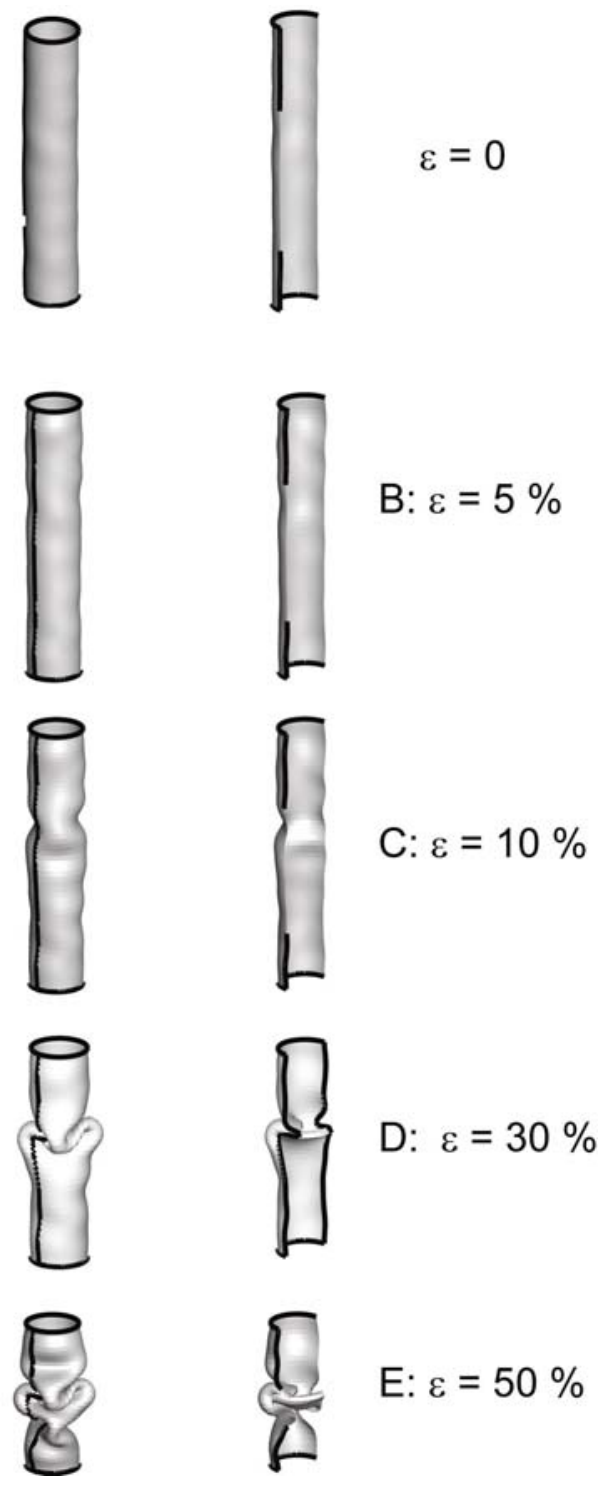

Figure 17. Response of a slender $90^{\circ}$ hollow truss to compression at $100 \mathrm{~m} / \mathrm{s}$. Note the absence of global buckling because of the inertial stabilization. The left figures show the deformations of the full tube. The right figures show mid-plane sections through the tube that reveal additional detail. 


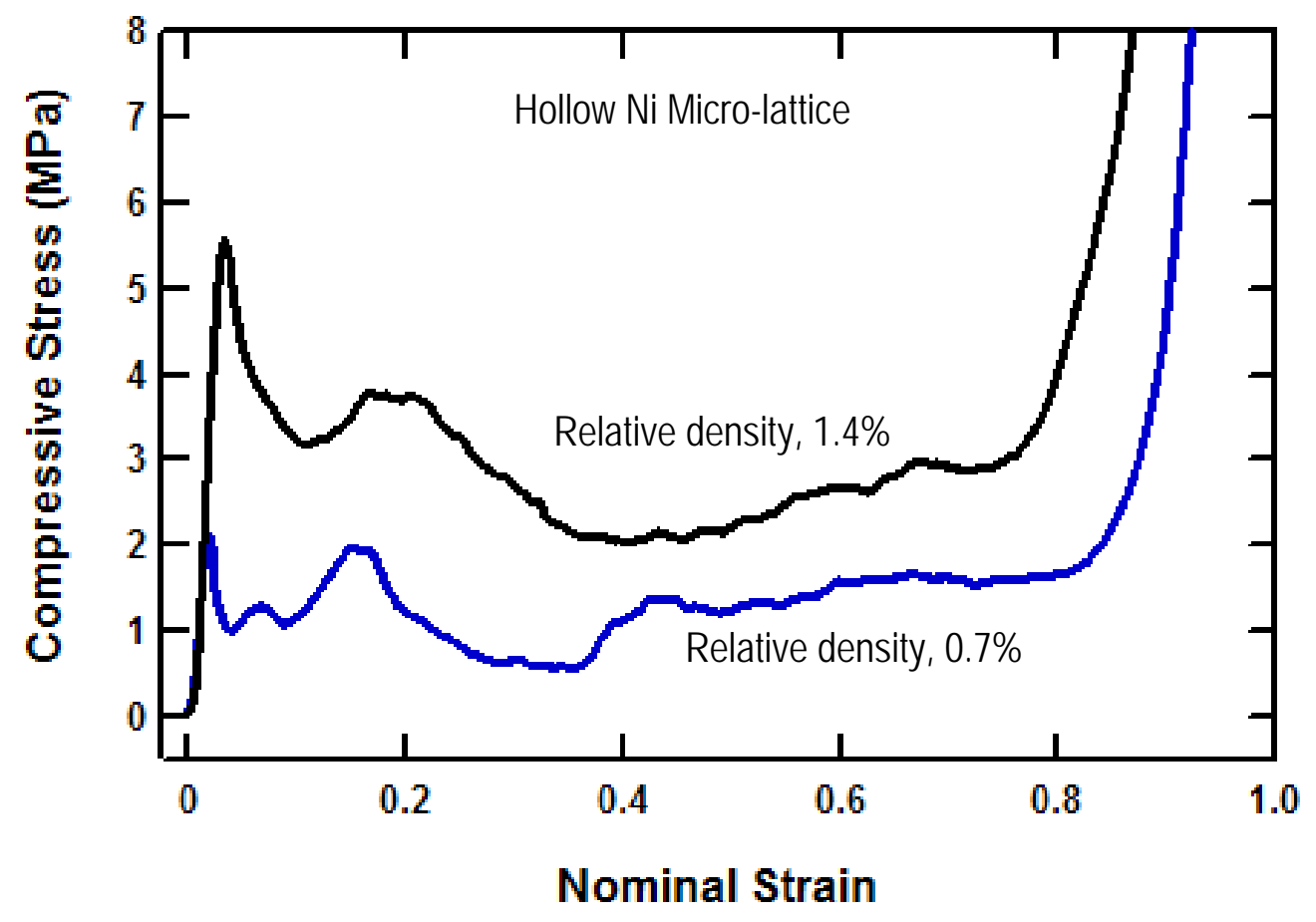

Figure 18. Experimental measurements for a Ni hollow truss micro-lattice (figure 2b)
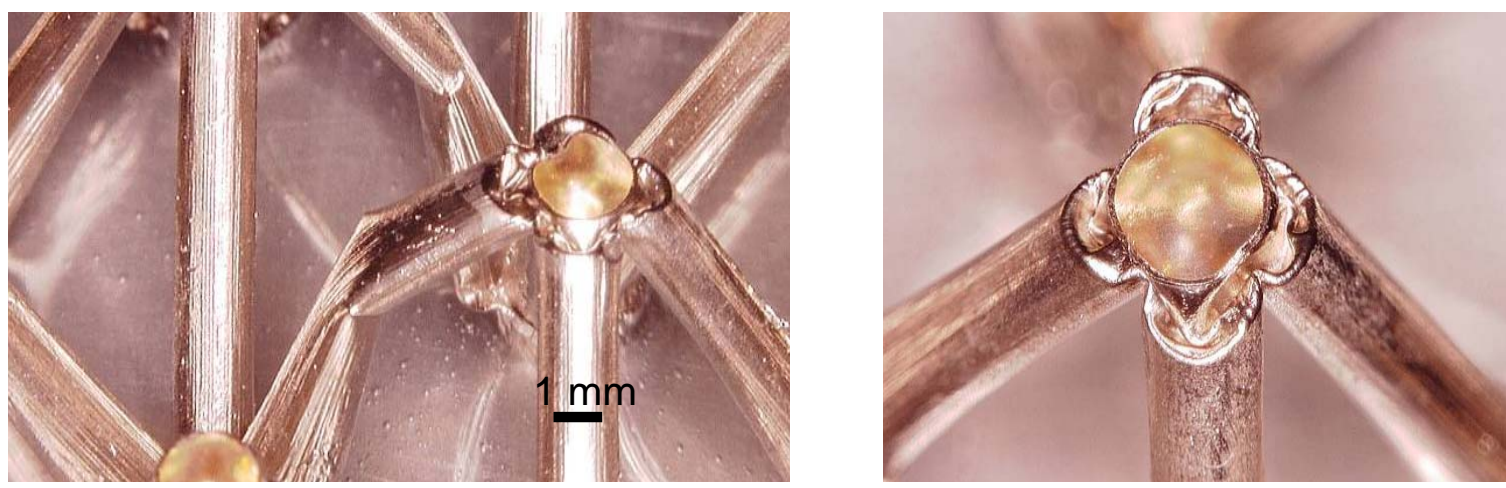

Figure 19. The deformation modes in the Ni hollow truss micro-lattice at $20 \%$ compression. (Left) Some members exhibit lateral buckling in the manner predicted by the simulations (figure 16). (Right) Other members exhibit a concertina-wrinkling mode at the nodes but otherwise remain straight. This mode is attributed to a larger R/t near the nodes and is suspected as the source of the substantial extra energy dissipation. 

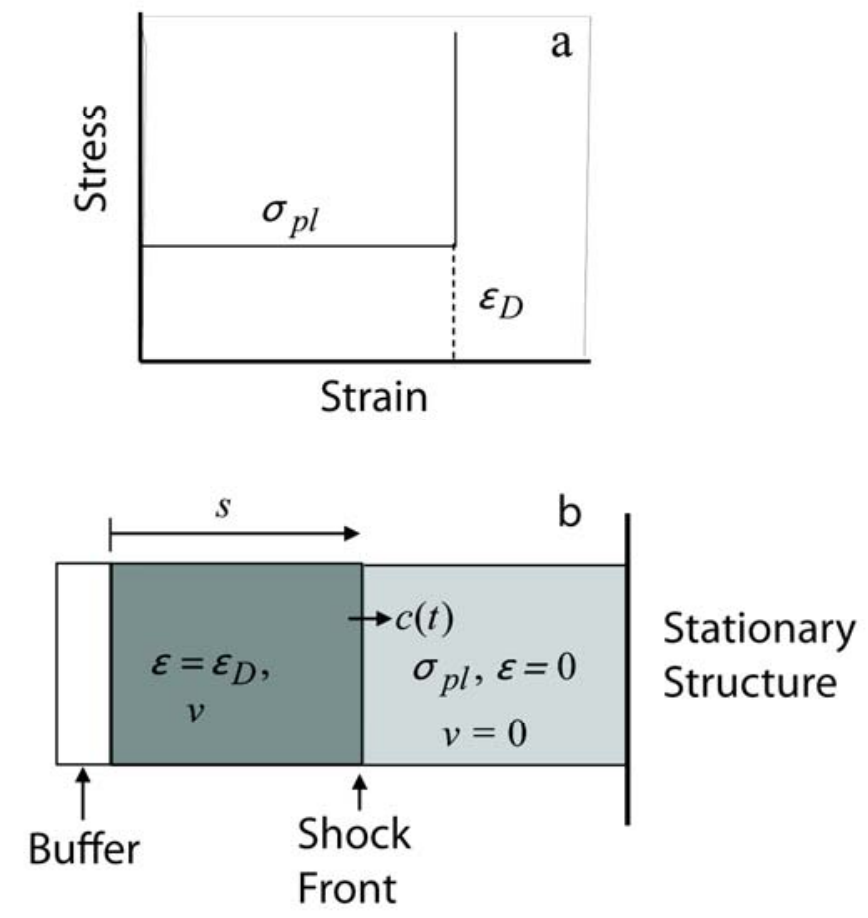

Figure A1. (a) The stress/strain curve for the cellular medium. (b). The material state upstream and downstream from the shock front. 